ARTICLE

https://doi.org/10.1038/s41467-019-08429-1

\title{
Zinc regulates ERp44-dependent protein quality control in the early secretory pathway
}

Satoshi Watanabe (1) 1, Yuta Amagai (1) 1, Sara Sannino ${ }^{2,4}$, Tiziana Tempio², Tiziana Anelli², Manami Harayama', Shoji Masui ${ }^{1}$, Ilaria Sorrentino ${ }^{2}$, Momo Yamada ${ }^{1}$, Roberto Sitia ${ }^{2}{ }^{2}$ \& Kenji Inaba ${ }^{1,3}$

Zinc ions $\left(\mathrm{Zn}^{2+}\right)$ are imported into the early secretory pathway by Golgi-resident transporters, but their handling and functions are not fully understood. Here, we show that $\mathrm{Zn}^{2+}$ binds with high affinity to the $\mathrm{pH}$-sensitive chaperone ERp44, modulating its localization and ability to retrieve clients like Erol $\alpha$ and ERAP1 to the endoplasmic reticulum (ER). Silencing the $\mathrm{Zn}^{2+}$ transporters that uptake $\mathrm{Zn}^{2+}$ into the Golgi led to ERp44 dysfunction and increased secretion of Ero1 $\alpha$ and ERAP1. High-resolution crystal structures of $\mathrm{Zn}^{2+}$-bound ERp44 reveal that $\mathrm{Zn}^{2+}$ binds to a conserved histidine-cluster. The consequent large displacements of the regulatory $\mathrm{C}$-terminal tail expose the substrate-binding surface and RDEL motif, ensuring client capture and retrieval. ERp44 also forms $\mathrm{Zn}^{2}+$-bridged homodimers, which dissociate upon client binding. Histidine mutations in the $\mathrm{Zn}^{2+}$-binding sites compromise ERp44 activity and localization. Our findings reveal a role of $\mathrm{Zn}^{2+}$ as a key regulator of protein quality control at the ER-Golgi interface.

\footnotetext{
${ }^{1}$ Institute of Multidisciplinary Research for Advanced Materials, Tohoku University, Sendai 980-8577, Japan. ${ }^{2}$ Division of Genetics and Cell Biology, VitaSalute University, IRCCS Ospedale San Raffaele, 20132 Milan, Italy. ${ }^{3}$ Core Research for Evolutional Science and Technology (CREST), Japan Science and Technology Agency (JST), Kawaguchi 332-0012, Japan. ${ }^{4}$ Present address: Department of Biological Sciences, University of Pittsburgh, A320 Langley Hall, 4249 Fifth Ave, Pittsburgh, PA 15260, USA. These authors contributed equally: Satoshi Watanabe, Yuta Amagai, Sara Sannino. These authors jointly supervised this work: Roberto Sitia, Kenji Inaba. Correspondence and requests for materials should be addressed to R.S. (email: sitia.roberto@hsr.it) or to K.I. (email: kinaba@tagen.tohoku.ac.jp)
} 
Z inc ions $\left(\mathrm{Zn}^{2+}\right)$ are essential cofactors for a variety of proteins $^{1,2}$. The metal ions serve as enzyme catalysts or as cofactors stabilizing the three-dimensional structures of proteins ${ }^{3-5}$. Moreover, free $\mathrm{Zn}^{2+}$ can also act as a second messenger in signal transduction ${ }^{6-8}$. Two families of transporters, ZnT (zinc transporter, SLC30) and ZIP (Zrt/Irt-like protein, SLC39), mediate $\mathrm{Zn}^{2+}$ homeostasis in cells ${ }^{9-12}$. The human genome contains $9 \mathrm{ZnT}$ and $14 \mathrm{ZIP}$ proteins with different tissue and subcellular distribution ${ }^{12}$. ZIP members mediate $\mathrm{Zn}^{2+}$ import into the cytosol, whereas members of the $\mathrm{ZnT}$ family conduct its efflux from the cytosol into intracellular compartments or to the outside of the cell. In particular, ZnT5, 6, 7, and 10 are known to import $\mathrm{Zn}^{2+}$ into the Golgi ${ }^{11}$, where the metal can be incorporated into secretory metalloenzymes ${ }^{13-19}$. The abundance and localization of ZnTs and ZIPs in the early secretory pathway (ESP) are consistent with the fundamental role of $\mathrm{Zn}^{2+}$ in regulating the structure and function of many secretory proteins. However, how the metal is handled in ESP remains to be understood.

ERp44, a chaperone of the protein disulfide isomerase (PDI) family, cycles between the ER and cis-Golgi compartments to patrol proteins that pass through the $\mathrm{BiP}$ and Calnexin/Calreticulin checkpoints ${ }^{20}$. This chaperone controls the traffic and oligomeric assembly of various secretory proteins, including IgM and adiponectin ${ }^{20,21}$. ERp44's clients also include several ERresident enzymes that lack an ER-retention motif, such as Erola, Prx4, FGE/SUMF1, and ERAP122-26. ERp44 traps its clients in the Golgi, retrieving them to the ER via KDEL receptors (KDELR $)^{27-31}$. ERp44 is regulated by the ER-Golgi pH-gradient, which enables the protein to capture clients in the weakly acidic post-ER compartments and release them in the $\mathrm{pH}$-neutral $\mathrm{ER}^{32}$.

The overall structure of ERp44 consists of three thioredoxin (Trx)-like domains ( $\mathbf{a}, \mathbf{b}$, and $\left.\mathbf{b}^{\prime}\right)$ and a flexible C-terminal tail (Ctail) that terminates with a RDEL motif ${ }^{33}$. The high-resolution crystal structures of ERp44 at $\mathrm{pH} 6.5$ and $7.2^{34}$ revealed that protonation of key histidine and cysteine residues induces $\mathrm{pH}$ dependent conformational changes in ERp44, promoting the exposure of the positively charged client-binding site and allowing the essential cysteine (Cys29) to form intermolecular disulfide bonds with clients of thiol-mediated quality control $^{35,36}$. Five conserved histidines located at the border between the $\mathbf{b}^{\prime}$ domain and the C-tail also have key roles in regulating the subcellular localization and client retention ability of ERp $44^{37}$. Of note, the high-resolution structure of ERp44 revealed that the three most highly conserved histidines (His299, His328, His332) form a histidine-clustered site (His-cluster) seemingly suitable for metal binding ${ }^{34}$. Taking into account the localization of several $\mathrm{Zn}^{2+}$ transporters in the ESP and the putative metal-binding site of ERp44, we surmised that $\mathrm{Zn}^{2+}$ regulates the function of this chaperone.

Here, we demonstrate that indeed $\mathrm{Zn}^{2+}$ binds to ERp44 with submicromolar affinity, dynamically controlling its traffic and activity. The crystal structure of $\mathrm{Zn}^{2+}$-bound ERp44 has established the structural basis of the $\mathrm{Zn}^{2+}$-mediated functional regulation of ERp44. Our findings identify a $\mathrm{Zn}^{2+}$-dependent mechanism of protein quality control at the ER-Golgi interface, revealing yet another physiological role for this essential metal ion in the secretory pathway.

\section{Results}

$\mathrm{Zn}^{2+}$ binds to ERp44 to induce its conformational changes. Considering that histidines can coordinate $\mathrm{Zn}^{2+}$, the presence of the highly conserved His-cluster at the regulatory C-tail (Fig. 1a) suggested that ERp44 binds $\mathrm{Zn}^{2+}$ via this cluster. To test this possibility, we first investigated whether and how ERp44 is able to bind $\mathrm{Zn}^{2+}$ by isothermal titration calorimetry (ITC) (Fig. $1 \mathrm{~b}$ and Supplementary Fig. 1A). ITC analyses showed that ERp44 binds 1.5 molar equivalents of $\mathrm{Zn}^{2+}$ with apparent $K_{\mathrm{d}}$ values of $135-295 \mathrm{nM}$ at $\mathrm{pH} 7.2-6.2$. This stoichiometry suggests that two ERp44 molecules, each of which binds one $\mathrm{Zn}^{2+}$ at the Hiscluster, coordinate another $\mathrm{Zn}^{2+}$ to form a homodimer. To confirm the $\mathrm{Zn}^{2+}$-dependent dimerization of ERp44, we performed size exclusion chromatography (SEC) analyses. When a mixture of ERp44 $(60 \mu \mathrm{M})$ and $\mathrm{ZnCl}_{2}(120 \mu \mathrm{M})$ was loaded onto a SEC column, the majority of ERp44 was eluted as non-covalent homodimers (Fig. 1c) with a molecular mass of $\sim 80 \mathrm{kDa}$, as determined by SEC combined with multi-angle light scattering (SEC-MALS) analyses (Supplementary Fig. 1C). The $\mathrm{Zn}^{2+}$-mediated dimerization was also observed within a $\mathrm{pH}$ range of 6.2-7.2 (Supplementary Fig. 1B).

Next, we investigated the influence of $\mathrm{Zn}^{2+}$ binding on the structure of ERp44 by far-UV CD and fluorescence spectroscopy. The far-UV CD spectra were insensitive to $\mathrm{Zn}^{2+}$ addition (Supplementary Fig. 1C), indicating that the secondary structure was maintained in ERp44 regardless of $\mathrm{Zn}^{2+}$ availability. By contrast, fluorescence spectroscopy using 1-anilinonaphthalene8 -sulfonate (ANS) revealed that $\mathrm{Zn}^{2+}$ binding induced significant conformational changes in ERp44 (Fig. 1d). In fact, similarly to the $\mathrm{pH}$ change from 7.2 to $6.2^{32}$, addition of excess $\mathrm{Zn}^{2+}$ strikingly increased the fluorescence intensity of ANS at both $\mathrm{pH} 6.2$ and $\mathrm{pH} 7.2$ (Fig. 1d, compare right and left panels), implying increased exposure of hydrophobic surfaces upon $\mathrm{Zn}^{2+}$ binding to ERp44. Subsequent chelation of $\mathrm{Zn}^{2+}$ with EDTA fully abolished this effect, highlighting the reversibility of the $\mathrm{Zn}^{2+}$-dependent conformational changes of ERp44. These results unequivocally demonstrate that $\mathrm{Zn}^{2+}$ reversibly binds to ERp44 with submicromolar affinity, inducing significant conformational changes and dimerization.

$\mathrm{Zn}^{2+}$ regulates the subcellular localization of ERp44. We previously showed that whereas wild-type ERp44 localizes in the ER-Golgi intermediate compartment (ERGIC) and cis-Golgi ${ }^{28}$, its mutants lacking one or all histidines of the conserved cluster can proceed further along the secretory pathway and be in part secreted out ${ }^{37}$. Thus, we surmised that $\mathrm{Zn}^{2+}$ binding to the Hiscluster of ERp44 could influence its intracellular traffic. To test this hypothesis, we examined the effects of $\mathrm{Zn}^{2+}$ depletion and replenishment on the subcellular localization of ERp44 by immunofluorescence. In untreated $\mathrm{HeLa}$ cells, endogenous ERp44 co-localized mainly with markers of the ERGIC and cisGolgi, but not of the early or late endosome, as observed previously $^{28}$ (Fig. 1e, left panels and Supplementary Fig. 2). When cells were treated for $30 \mathrm{~min}$ with a membrane-permeable $\mathrm{Zn}^{2+}$ chelator, $N, N, N^{\prime}, N^{\prime}$-tetrakis (2-pyridylmethyl) ethylenediamine (TPEN), ERp44 accumulated in a perinuclear area, largely colocalizing with GM130, a cis-Golgi marker (Fig. 1e, middle panels, and $1 \mathrm{f}$ ). Subsequent addition of excess $\mathrm{ZnSO}_{4}$ was sufficient to re-localize endogenous ERp44 primarily to the ERGIC (Fig. 1e, right panels and 1f). Similar phenomena were observed for endogenous ERp44 in HepG2 cells (Supplementary Fig. 3A) and for YFP-tagged ERp44 (YFP-ERp44) overexpressed in HeLa cells (Supplementary Fig. 3B, C).

Time-lapse fluorescence imaging allowed to dynamically visualize the $\mathrm{Zn}^{2+}$-dependent movements of ERp44 in living cells (Fig. 1g, Supplementary Movies 1 and 2). After $30 \mathrm{~min}$ in the presence of TPEN, YFP-ERp44 accumulated strikingly in a perinuclear GM130-positive area, and returned to the ER within 15 min after addition of zinc pyrithione (ZPT; $20 \mu \mathrm{M})$, a $\mathrm{Zn}^{2+}$-specific ionophore (Fig. 1g, lower panel, Supplementary Movies 1 and 2). The overall structure of the Golgi was not 
a

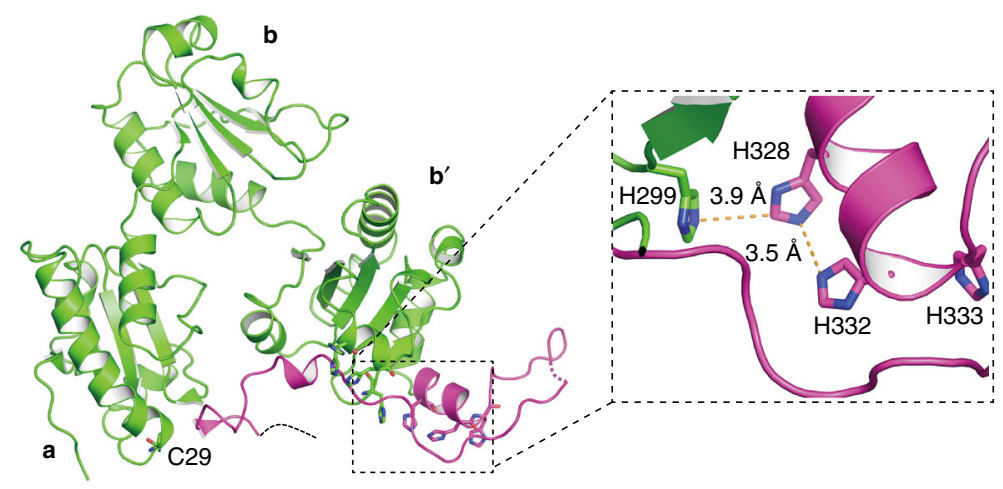

b

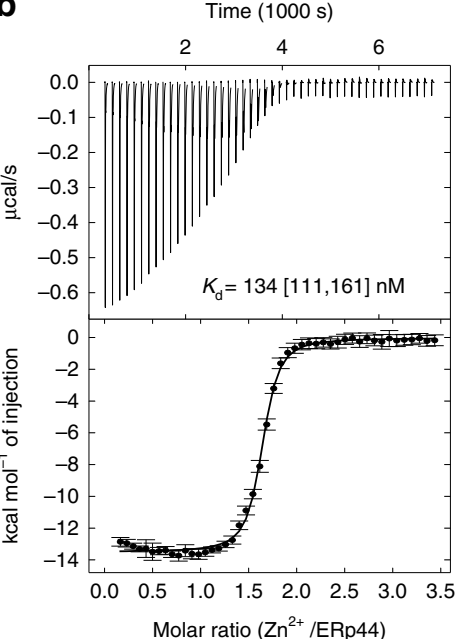

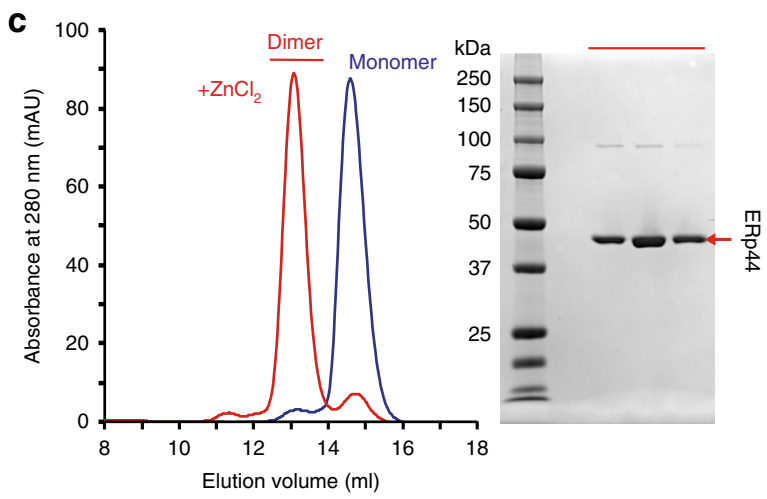

d
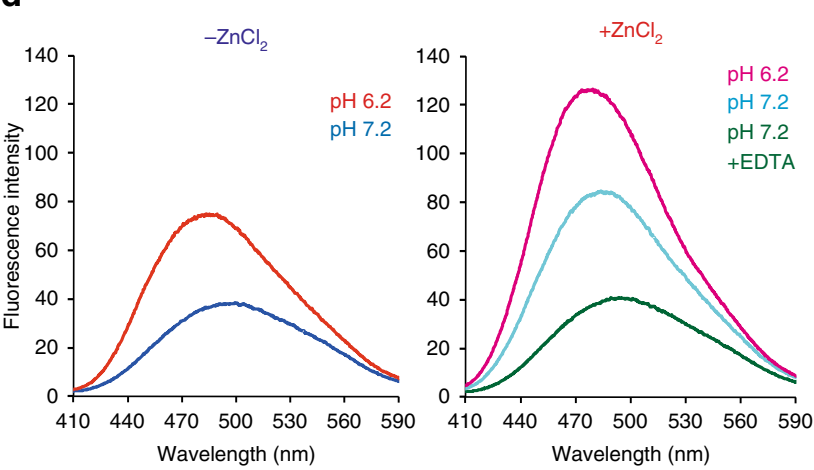

e

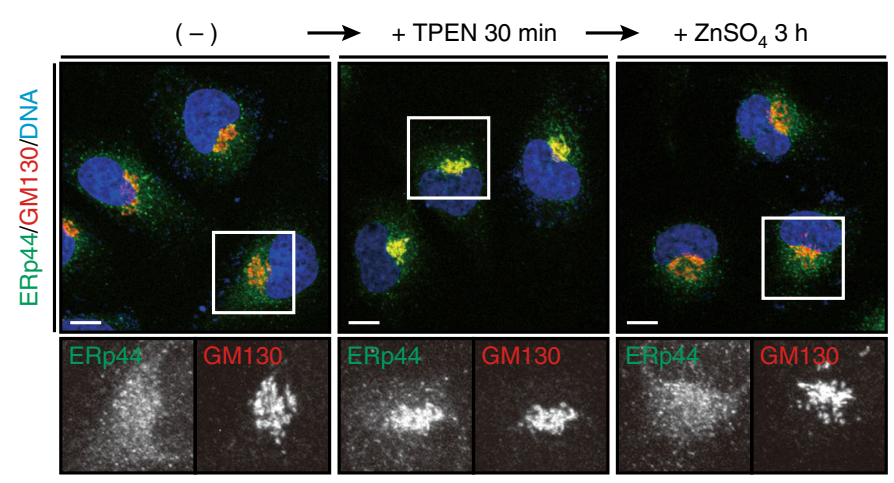

f
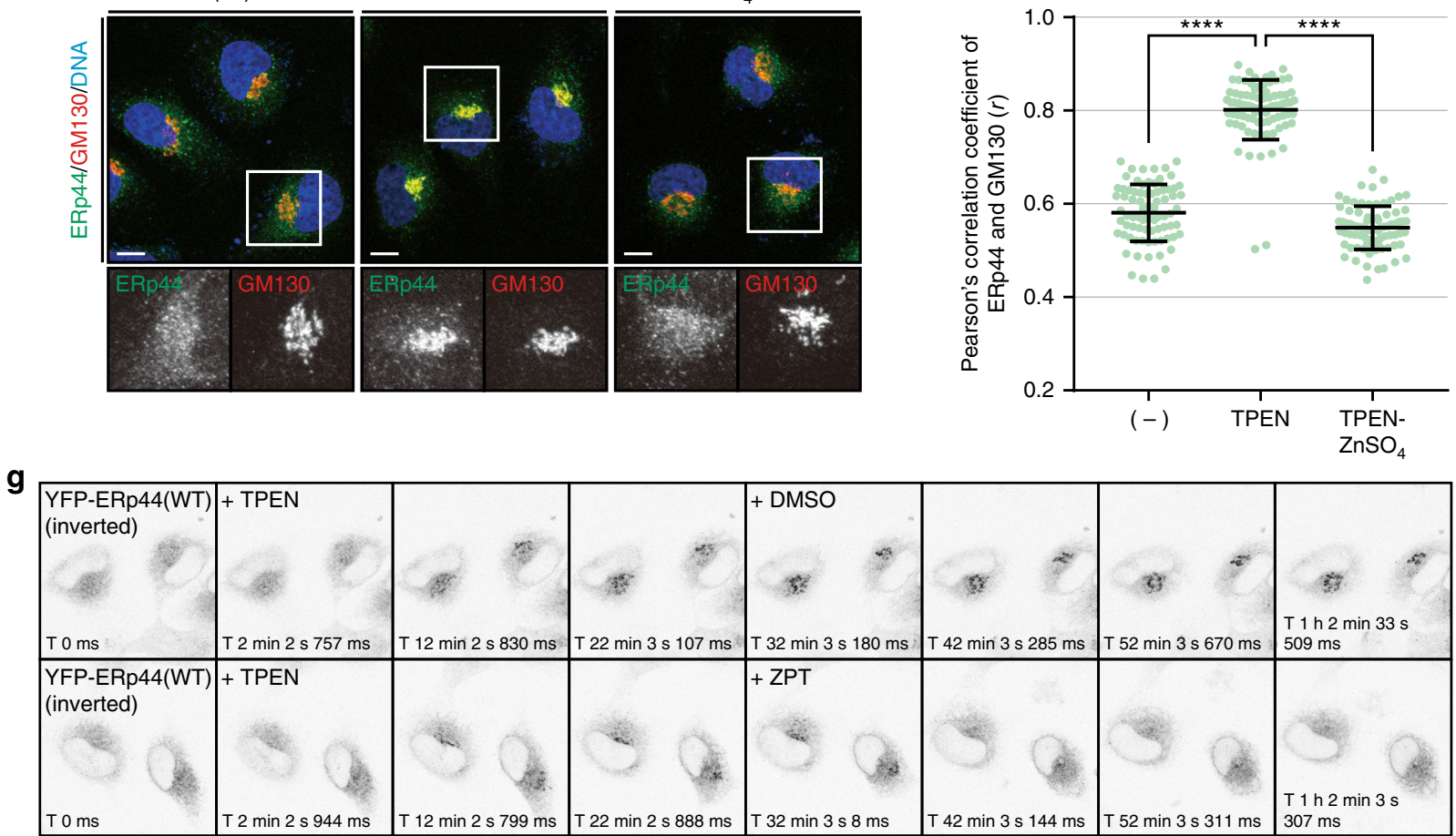

significantly affected by short-time treatments with TPEN and ZPT (Supplementary Fig. 2B, lower panels), underlining the specificity of the phenomena observed for ERp44. Additionally, DMSO, a reagent used to dissolve TPEN and ZPT, hardly altered ERp44 localization (Fig. 1g, upper panel; Supplementary Movie 2). Taken together, the above results demonstrate that $\mathrm{Zn}^{2+}$ dynamically and reversibly controls the intracellular traffic of ERp44. 
Fig. $1 \mathrm{Zn}^{2}+$ binds to ERp44, affecting its structure and localization. a Updated crystal structure of ERp44 at 2.0 A resolution (PDB ID: 5GU6). The three Trxlike domains ( $\mathbf{a}, \mathbf{b}$, and $\mathbf{b}^{\prime}$ ) and C-tail are shown in green and magenta, respectively. The inset shows a close-up view of the His-cluster composed of His299, His328, and His332. b ITC raw data (upper) and binding isotherm data (lower) for titration of $\mathrm{ZnCl}_{2}(500 \mu \mathrm{M})$ into $\mathrm{ERp} 44$ (30 $\left.\mu \mathrm{M}\right)$ at pH 7.2. Bars represent the errors in the peak integration for each injection estimated by NITPIC 72 . The global analysis was performed to estimate the $K_{\mathrm{d}}$ and $\Delta H$ values with SEDPHAT ${ }^{73}$ assuming 1:1 binding. The apparent $K_{\mathrm{d}}$ is shown with $68.3 \%$ confidence interval in brackets. c SEC analysis of ERp44 (60 $\left.\mu \mathrm{M}\right)$ in the presence (red line) or absence (blue line) of $\mathrm{Zn}^{2+}$ ions $(120 \mu \mathrm{M})$. Peak fractions of the ERp $44-\mathrm{ZnCl}_{2}$ mixture eluted at $12.5-14.0 \mathrm{~mL}$ were analyzed by nonreducing SDS-PAGE (right), suggesting that major portion of ERp44 forms non-covalent homodimers in a $\mathrm{Zn}^{2+}$-dependent manner. $\mathbf{d}$ ANS fluorescence spectra were measured for ERp44 at pH 7.2 or 6.2 in the absence (left) or presence (right) of $\mathrm{ZnCl}_{2}$. The higher and blue-shifted fluorescence peaks observed at lower $\mathrm{pH}$ and those in the presence of $\mathrm{Zn}^{2+}$ suggest the exposure of hydrophobic surfaces in ERp44. Subsequent addition of EDTA to the ERp44- $\mathrm{ZnCl}_{2}$ mixture returned the fluorescence to the initial level. e Confocal immunofluorescence images showing the intercellular localization of endogenous ERp44 (in green) in HeLa cells. Cells were co-stained with an antibody to GM130 (in red) and with DAPI (in blue) to highlight the Golgi and nucleus, respectively. Note that ERp44 accumulates in the Golgi upon $\mathrm{Zn}^{2+}$ deprivation and returns to the ER upon $\mathrm{Zn}^{2+}$ replenishment. Scale bars, $10 \mu \mathrm{m}$. f Quantitative analyses of the Pearson's correlation coefficients for the co-localization of endogenous ERp44 with GM130 based on the immunofluo rescence images shown in $\mathbf{e}$. Dots indicate individual data points ( $\geq 70$ cells for each condition). Bars indicate the means \pm SD. ${ }^{\star \star \star \star} p<0.0001$. $\mathbf{g}$ Time-lapse fluorescence imaging of YFP-ERp44 in living cells. HeLa cells transfected with YFP-ERp44 were treated with TPEN for 30 min and then with ZPT or DMSO (vehicle) for $30 \mathrm{~min}$. As described for endogenous ERp44, $\mathrm{Zn}^{2+}$ depletion and replenishment alters intracellular localization of YFP-ERp44 in a reversible manner

$\mathrm{Zn}^{2+}$ at physiological levels controls ERp44 traffic and activity. To further investigate the physiological role of $\mathrm{Zn}^{2+}$ in ERp44 regulation, we silenced the Golgi-resident transporters ZnT5, ZnT6, and ZnT7. Their knockdown was recently reported to cause secretion of inactive apo- $\mathrm{Zn}^{2+}$-enzymes, in line with their pivotal roles in $\mathrm{Zn}^{2+}$ uptake into the ESP ${ }^{19}$. Upon $\mathrm{ZnT5/6/7}$ triple knockdown (Supplementary Fig. 4E), endogenous ERp44 co-localized with GM130 (Fig. 2a, b), as observed in TPENtreated cells (Fig. 1e, Supplementary Fig. 2A, B). Similarly, endogenous ERp44 in HepG2 cells (Supplementary Fig. 4A, B and G) and overexpressed YFP-ERp44 in HeLa cells (Supplementary Fig. 4C and D) moved to the cis-Golgi upon ZnT5/6/ 7 silencing. Thus, ERp44 predominantly accumulates in the Golgi upon $\mathrm{ZnT5/6/7}$ triple knockdown, indicating that physiological levels of $\mathrm{Zn}^{2+}$ in the ESP regulate its subcellular localization.

Next, we examined the effects of $\mathrm{Zn}^{2+}$ depletion on the ability of ERp44 to retain overexpressed ERAP1 and Erola, two enzymes that normally reside in the ER despite the lack of a KDEL-motif ${ }^{20,25,27}$. Compared to controls, TPEN treatment enhanced secretion of these two clients (Fig. 2c, lanes 1-4). Importantly, ZnT5/6/7 triple knockdown yielded similar results (Fig. 2c, lanes 5 and 6, Supplementary Fig. 4F), strongly suggesting that $\mathrm{Zn}^{2+}$ imported into post-ER compartments via one or more $\mathrm{Zn}^{2+}$ transporter(s) have an essential role in efficient client retention by ERp44. In contrast, the ER-resident chaperone PDI was not secreted, confirming the specificity of the effects of $\mathrm{Zn}^{2+}$ deprivation on ERp44 (Fig. 2c, bottom). The consequences of $\mathrm{Zn}^{2+}$ deprivation were more evident for ERAP1 than for Erola (Fig. 2d), probably because Erola is also retained in the ER by interactions with its redox partner $\mathrm{PDI}^{22,24,38,39}$. Accordingly, the subcellular localization of Erol $\alpha$ molecules that were not secreted was unaltered by $\mathrm{Zn}^{2+}$ depletion and replenishment (Supplementary Fig. 4H). Moreover, we observed that Erola itself has no ability to bind $\mathrm{Zn}^{2+}$ (Supplementary Fig. 4I).

To confirm the specific binding of ERp44 to $\mathrm{Zn}^{2+}$, we tested the ability of ERp44 to bind copper $\left(\mathrm{Cu}^{2+}\right)$ and manganese $\left(\mathrm{Mn}^{2}\right.$ ${ }^{+}$) ions, because copper transporters and manganese transporters are known to be localized in the secretory pathway ${ }^{40,41}$. The ITC analysis showed that ERp44 binds $\mathrm{Cu}^{2+}$ ions, but with micromolar affinity $\left(K_{\mathrm{d}}=1.65 \mu \mathrm{M}\right)$ (Supplementary Fig. $5 \mathrm{~A}$ ). No interactions were detected between ERp44 and $\mathrm{Mn}^{2+}$ (Supplementary Fig. 5B). Notably, copper chelation by tetraethylenepentamine little affected the subcellular localization of ERp44 (Supplementary Fig. 5C-E), suggesting that copper is not involved in ERp44 regulation. Altogether, we conclude that $\mathrm{Zn}^{2+}$ at physiological levels specifically controls the cellular traffic and client retention activity of ERp44.

Structure of the $\mathbf{Z n}^{2+}$-bound form of ERp44. To reveal how $\mathrm{Zn}^{2+}$ impacts ERp44 conformation at atomic resolution, we determined the crystal structure of the ERp44- $\mathrm{Zn}^{2+}$ complex at $2.45 \AA$ resolution by the single anomalous dispersion (SAD) method using the anomalous signals of $\mathrm{Zn}^{2+}$ (Supplementary Fig. 6A, B, and Supplementary Tables 5 and 6). The crystallographic asymmetric unit contains four ERp44 molecules, which form two homodimers (dimer 1: Mol A and Mol B; dimer 2: Mol $\mathrm{C}$ and Mol D) (Supplementary Fig. 6A). The overall architecture of the $\mathrm{Zn}^{2+}$-bound ERp44 homodimer resembles two clover leafs aligned tail to tail (Fig. 3a). The two ERp44 monomers in the homodimer are nearly related by a twofold axis (Fig. 3a top, a vertical line). At the center of the dimer, the $\alpha 12$ helices in the $\mathbf{b}^{\prime}$ domains are bridged by a $\mathrm{Zn}^{2+}$ ion (Fig. 3b, left). The $\mathrm{Zn}^{2+}$-bridged non-covalent homodimer is consistent with the results of the ITC and SEC analyses (Fig. 1b, c). Within the homodimer, larger dimer interfaces are also formed between a part of the C-tail of one protomer and the a domain of the other protomer, including a $\pi-\pi$ stacking interaction between His333 $(\mathrm{Mol} \mathrm{A})$ and Phe31 ( $\mathrm{Mol} \mathrm{B})$, an arginine stacking interaction between Arg329 (Mol A) and Arg30 (Mol B) and several hydrogen bonds and van der Waals contacts between the C-tail segment (residues Ala350-Glu356) in Mol A and a part of the a domain (residues Lys77 and Arg95 to Arg98) in Mol B (Fig. 3b, right).

Unlike metal-free ERp44, the $\mathrm{Zn}^{2+}$-bound ERp44 monomer adopts an open conformation in which the C-tail is released from the a domain and the client-binding surface including Cys29 is exposed to the solvent (Fig. 3c). By contrast, the C-tail is closed to mask Cys29 and its neighboring region in metal-unbound ERp44 (Fig. 3c, right $)^{34}$. The C-terminal region (residues 359-378) of each protomer in the $\mathrm{Zn}^{2+}$-bound homodimer shows very high B-factors, adopting different conformations (Supplementary Fig. 6C, D). Residues 366-377 of Mol A insert into the interior of the dimer interface (Supplementary Fig. 6E), whereas the residues 360-366 of Mol B extend toward outside the molecule (Supplementary Fig. 6F). The corresponding regions of Mol C and Mol D are more disordered (Supplementary Fig. 6D). Thus, the C-terminal region seems to partially stabilize the dimeric structure, but its contribution may vary in different protomers. 
a

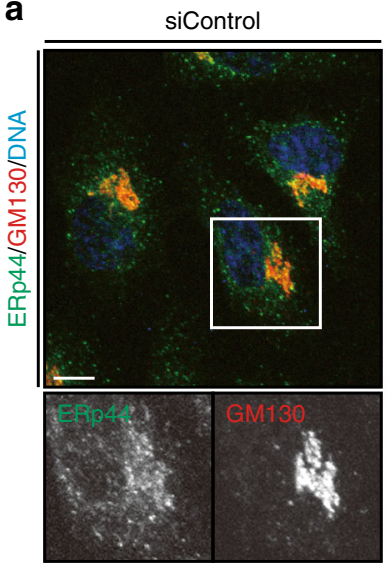

siZnT5/6/7

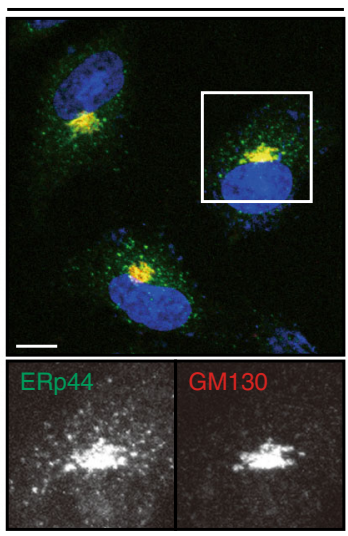

C

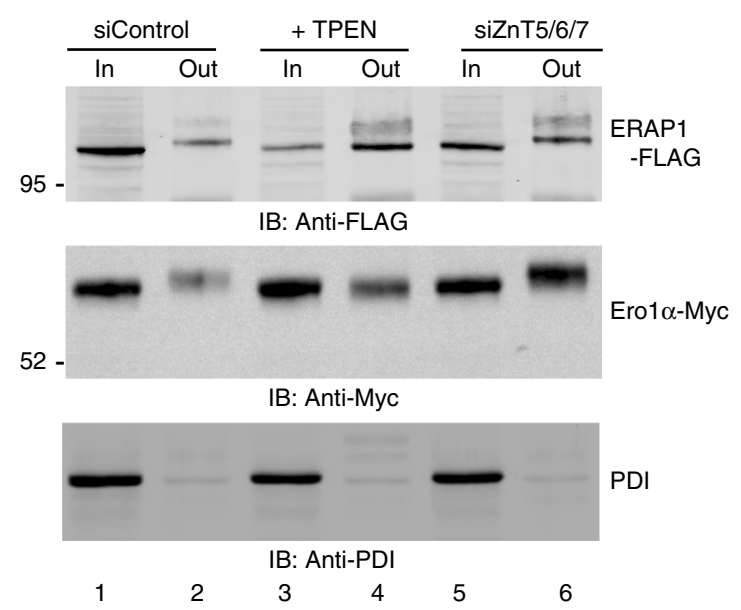

d

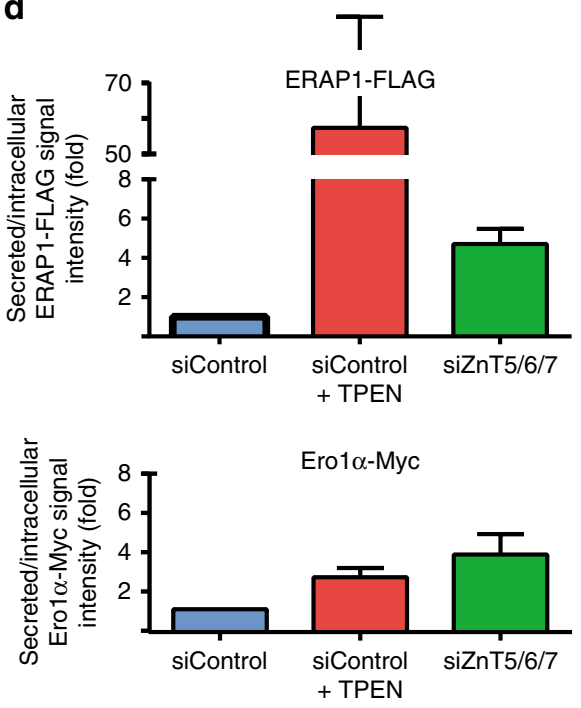

Fig. 2 Knockdown of the Golgi-resident $\mathrm{Zn}^{2+}$ transporters alters ERp44 localization and function in cells. a Confocal immunofluorescence images showing the intracellular localization of ERp44 after ZnT5, 6 and 7 triple knockdown (siZnT5/6/7) or treatment with control siRNA (siControl). Cells were costained with antibodies specific for ERp44 (in green), GM130 (in red) and DAPI (in blue). Scale bar, $10 \mu m$. The simultaneous knockdown of ZnT5/6/7 caused accumulation of ERp44 in the Golgi, similarly to TPEN (Fig. 1e). b Quantitative analysis of Pearson's correlation coefficients of the co-localization of endogenous ERp44 with GM130 based on the immunofluorescence images shown in a. Dots indicate individual data points ( $\geq 80$ cells for each condition, unpaired $t$-test). Bars indicate the means \pm SD. ${ }^{\star \star \star \star} p<0.0001$. c, d TPEN and ZnT5/6/7 triple knockdown promote secretion of two ERp44 clients, Ero1 $\alpha$ and ERAP1, but not of PDI. Lysates of HeLa transfectants (In) and TCA-precipitated culture supernatants (Out) were resolved by SDS-PAGE under reducing conditions and blot membranes were decorated with anti-FLAG, anti-Myc, and anti-PDI antibodies to visualize ERAP1, Ero1 $\alpha$, and PDI, respectively. To quantify retention efficiency, the ratios between the band intensity of the secreted and intracellular ERAP1 and Ero1 $\alpha$ were calculated and expressed as fold of induction relative to untreated cells (d). Bars indicate the means \pm SEM of five independent experiments. Clearly, depleting $Z \mathrm{n}^{2+}$ from the ESP by TPEN or ZnT5/6/7 triple knockdown allowed secretion of ERAP1 and Ero1 $\alpha$. Note that secreted ERAP1 and Ero1 $\alpha$ displayed slower gel electrophoretic mobility than intracellular ones, possibly due to further processing of N-glycans and/or O-glycosylation in the Golgi. No band shifts were observed for TPEN treatment, which may be explained by TPEN-triggered inactivation of Golgi-resident metal-dependent galactosyltransferases and/or glycosyltransferases

Three $\mathrm{Zn}^{2+}$-binding sites in ERp44. The present structure reveals three types of $\mathrm{Zn}^{2+}$ binding sites in the ERp44 homodimer (sites 1, 2, and 3), with a total of five $\mathrm{Zn}^{2+}$ ions aligned close to the dimer interface (Fig. 3a, right panels). Site 1 is formed by the His-cluster in each protomer. $\mathrm{Zn}^{2+}\left(\mathrm{Zn} 1\right.$ and $\left.\mathrm{Zn1}^{\prime}\right)$ is coordinated by three histidines (His299, His328 and His332) and a water molecule in a tetrahedral geometry (Fig. 3a, right top) (Supplementary Table 8). This mode of $\mathrm{Zn}^{2+}$ coordination is widely found in other $\mathrm{Zn}^{2+}$-binding proteins such as carbonic anhydrase II, insulin hexamers, and the serine protease tonine $e^{5,42,43}$. Site 1 is also stabilized by van der Waals contacts with several surrounding residues from the adjacent protomer in the $\mathrm{Zn}^{2+}$-bound homodimer (Supplementary Fig. 6G).

Site 2 is formed at the center of the dimer interface, contributing directly to homodimerization of ERp44. Two antiparallel histidine pairs (His277 and His288) in the mutually adjacent $\mathbf{b}^{\prime}$ domains coordinate a $\mathrm{Zn}^{2+}(\mathrm{Zn} 2)$ in a tetrahedral geometry (Fig. 3a, right middle) (Supplementary Table 8). Such intermolecular $\mathrm{Zn}^{2+}$ coordination by four histidines is found in a couple of proteins including the heme-responsive zinc-finger 

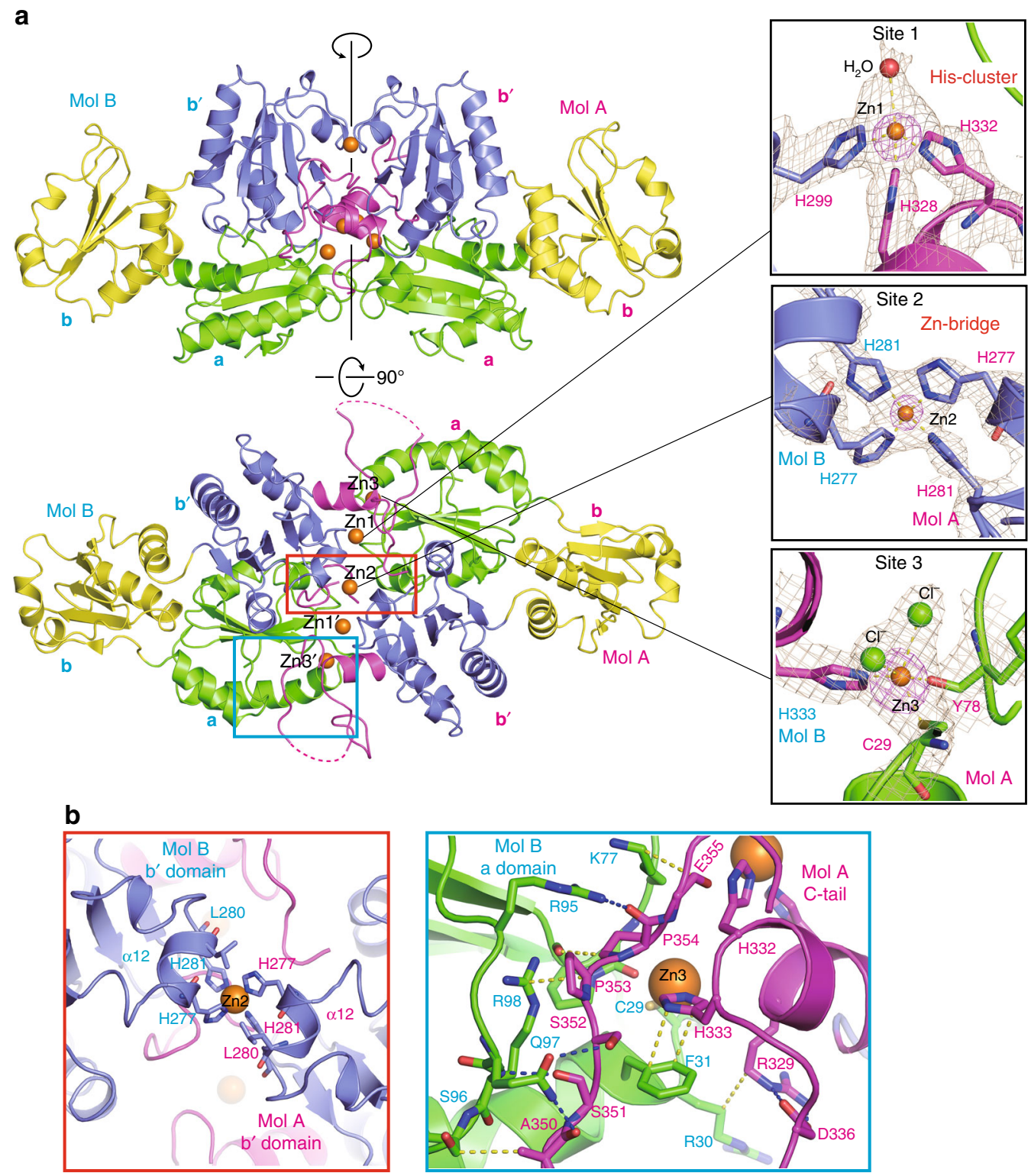

C
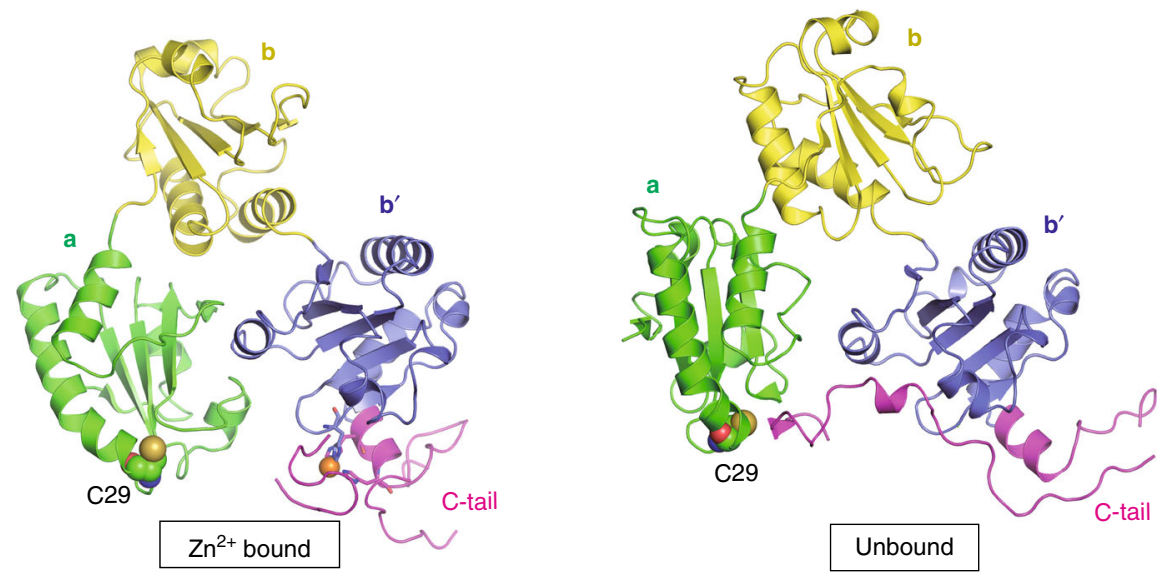

transcription factor $\mathrm{HAP} 1^{44}$ and the RNA-binding anti-termination protein $\mathrm{HutP}^{45}$.

Site 3 is formed at both the ends of the dimer interface (Fig. 3a, right bottom). $\mathrm{Zn}^{2+}$ at site $3(\mathrm{Zn} 3)$ is coordinated by the $\mathrm{N} \delta$ of His 333 of one protomer, the thiol group of Cys29 and the main- chain carbonyl oxygen of Tyr74 of the other protomer and two chloride ions (see also Methods) (Supplementary Table 8). To our knowledge, the trigonal bipyramid geometry of site 3 has not been found so far in any metalloproteins of known structure ${ }^{46}$. Indeed, carbonyl oxygen is a minor ligand for coordination of $\mathrm{Zn}^{2+4,47}$. 
Fig. 3 Structure of $Z n^{2+}$-bound form of ERp44. a Top and side view of the overall structure of the $Z n^{2+}$-bound dimer of ERp44. The $\mathbf{a}, \mathbf{b}, \mathbf{b}^{\prime}$ domains and Ctail of $\mathrm{Mol} \mathrm{A}$ and $\mathrm{Mol} \mathrm{B}$ are shown in green, yellow, blue and magenta, respectively. The $\mathrm{Zn}^{2+}$ ions are represented by orange spheres. A vertical black line represents a non-crystallographic twofold axis. The right insets display the close-up views of the three $\mathrm{Zn}^{2+}$ binding sites: site 1 (top), site 2 (middle) and site 3 (bottom). Simulated annealing 2Fo-Fc omit maps at 1-1.3 $\sigma$ and anomalous difference Fourier map at $15 \sigma$ are shown in brown and magenta, respectively. $\mathbf{b}$ Close-up views of the dimer interfaces; (left): highlighted view of the red box in $\mathbf{a}$, which illustrates interactions formed between the $\alpha 12$ helices of the $\mathbf{b}^{\prime}$ domains in ERp44 dimer; (right): highlighted view of the blue box in $\mathbf{a}$, which illustrates interactions formed between the C-tail of Mol A and the $\mathbf{a}$ domain of Mol B. Hydrogen bonds and van der Waals contacts are shown by blue and yellow dashed lines, respectively. c Comparison of the overall structure of the $\mathrm{Zn}^{2+}$-bound (left) and unbound (right) forms of the ERp44 protomer. The essential cysteine (Cys29) is shown as spheres

The presence of site 3 was unexpected, since our ITC analyses did not detect a third $\mathrm{Zn}^{2+}$ binding (Fig. 1b). The observation that the one-to-two mixture of ERp44 and $\mathrm{Zn}^{2+}$ eluted mostly as a $\mathrm{Zn}^{2+}$-bridged homodimer in the SEC analysis (Fig. 1c) suggests that $\mathrm{Zn}^{2+}$ binding to site 3 is not necessary for dimerization (see also the last paragraph of the next section).

Sequential $\mathrm{Zn}^{2+}$ binding to ERp44. To elucidate the molecular details of $\mathrm{Zn}^{2+}$-binding to ERp44, we constructed two kinds of His mutants in which either set of His277 and His281 (H277/ 281A), or His299, His328, and His332 (3HA, previously called $\mathrm{ABC}^{37}$ ) were replaced by Ala. Far-UV CD measurements indicated that both mutants maintained the overall secondary structure, independently of $\mathrm{Zn}^{2+}$ (Supplementary Fig. 7A, B). As expected, the H277/281A mutant, which lacks site 2, did not form $\mathrm{Zn}^{2+}$-dependent homodimers (Supplementary Fig. 7C, left). ITC analyses revealed that H277/281A bound one molar equivalent of $\mathrm{Zn}^{2+}$ (Supplementary Fig. 7D, left), which most likely reflects $\mathrm{Zn}^{2+}$ binding to site 1 only. In contrast, neither $\mathrm{Zn}^{2+}$-dependent dimerization nor large enthalpy changes were observed for the 3HA mutant, which retains site 2 but lacks the His-cluster (site 1) (Supplementary Fig. 7C, D, right). These results suggest that $\mathrm{Zn}^{2+}$ binding to site 1 is required for $\mathrm{Zn}^{2+}$ binding to site 2 and hence, for the formation of the $\mathrm{Zn}^{2+}$-bridged homodimer.

To further analyze $\mathrm{Zn}^{2+}$ binding to these two sites, we calculated the Scatchard plots based on the ITC data (Supplementary Fig. 8A). Due to the apparent single-phase ITC profiles (Fig. $1 \mathrm{~b}$ and Supplementary Fig. 1A), we could not determine the thermodynamic parameters for $\mathrm{Zn}^{2+}$ binding to the individual sites separately. However, the calculated Scatchard plots displayed concave downward curves at all $\mathrm{pH}$ measured (Supplementary Fig. 8A), a feature characteristic of multiple ligand binding with 'positive cooperativity' ${ }^{48,49}$. The Hill coefficients estimated from the saturation curve based on the ITC data were significantly higher than 1 (Supplementary Fig. 8B), supporting the positive cooperativity in sequential $\mathrm{Zn}^{2+}$ binding to ERp44. This means that the first $\mathrm{Zn}^{2+}$ binding to site 1 significantly increases the affinity for $\mathrm{Zn}^{2+}$ of site 2, facilitating the formation of the $\mathrm{Zn}^{2+}$-bridged dimer. In line with this, our SEC analyses demonstrated that significant amounts of $\mathrm{Zn}^{2+}$-mediated ERp44 dimers were generated even at ERp44/ $\mathrm{Zn}^{2+}$ ratios of 1:0.5 to $1: 1$, before the saturation of site 1 with $\mathrm{Zn}^{2+}$ (Supplementary Fig. 8C). In this context, dimerization appears to stabilize the $\mathrm{Zn}^{2+}$-binding configuration of site 1 via interactions with $\operatorname{Trp} 28$ and Ser76 of the other protomer (Supplementary Fig. 4G). To estimate the dissociation constant for the $\mathrm{Zn}^{2+}$-bridged dimer, we also performed the dissociation titration experiments (Supplementary Fig. 8D). However, no significant heat changes were observed during the dilution titration. Thus, the $\mathrm{Zn}^{2+}$-mediated ERp44 dimer hardly disassembles at a concentration of $0.1 \mu \mathrm{M}$ (100-fold dilution of titrant), implying that the dissociation constant for ERp44 dimerization is below $0.1 \mu \mathrm{M}$.
We also constructed a His333/Ala mutant (H333A) to investigate the role of site 3 (Supplementary Fig. 9). In the $\mathrm{Zn}^{2+}$-bridged dimer, His333 not only coordinates $\mathrm{Zn} 3$, but also forms a $\pi-\pi$ stacking interaction with Phe31, seemingly contributing to stabilization of the dimer. In agreement with this, the SEC analysis showed that $\mathrm{H} 333 \mathrm{~A}$ was eluted only slightly faster in the presence of $\mathrm{ZnCl}_{2}$, suggesting that this mutant did not form a stable homodimer with $\mathrm{Zn}^{2+}$ (Supplementary Fig. 9A). ITC analysis showed that $\mathrm{H} 333 \mathrm{~A}$ bound one $\mathrm{Zn}^{2+}$ per monomer, with much lower affinity $\left(K_{\mathrm{d}}=2.49 \mu \mathrm{M}\right)$ than WT and $\mathrm{H} 277 /$ 281/A (2HA) (Supplementary Fig. 9B). This may be explained by the likely perturbation of $\mathrm{H} 333 \mathrm{~A}$ mutation on the local conformation at site 1 involving His328 and His332. Thus, His333 seems to have auxiliary roles in $\mathrm{Zn}^{2+}$ binding to ERp44 and hence dimer formation.

$\mathrm{Zn}^{2+}$ binding induces striking conformational changes of ERp44. The above crystallographic studies further reveal that $\mathrm{Zn}^{2+}$ binding to site 1 induces striking movements of the C-tail, accompanied by significant rearrangements of the Trx-like domains (Supplementary Movie 3). In the metal-free state, Pro353 stacks with Phe331 and makes van der Waals contacts with His328, His299 and His332, intervening between these three histidines (Fig. 4a, left). Such separate location of the histidines involved does not seem optimal for $\mathrm{Zn}^{2+}$ binding. In the $\mathrm{Zn}^{2+}$-bound state, however, Pro353 is largely moved out and the a16 helix gets closer to His299 (Fig. 4a, middle and right). As a result, His299, His328, and His332 adopt a configuration suitable for $\mathrm{Zn}^{2+}$ binding (site 1). Noteworthy, the large movement of Pro353 accompanies a striking C-tail movement toward the $\mathbf{b}^{\prime}$ domain, disrupting the interactions between the $\mathrm{C}$-tail and the a domain (Fig. 3C). In this state, Cys29 becomes fully exposed to the solvent (Figs. 3c, 4b), and the $\mathbf{a}$ and $\mathbf{b}$ domains are rotated by $\sim 77^{\circ}$ with respect to the $\mathbf{b}^{\prime}$ domain (Supplementary Fig. 10A).

The $\mathrm{Zn}^{2+}$-dependent conformational changes also influence the molecular surface properties of ERp44. In the a domain, the surroundings of Cys29 are found to be positively charged to a significant extent (Fig. 4b, upper). The surface features of $\mathrm{Zn}^{2+}$-bound ERp44 protomer are similar to those observed in ERp44 at weakly acidic $\mathrm{pH}^{34}$. Additionally, a large hydrophobic patch between the $\mathbf{b}$ and $\mathbf{b}^{\prime}$ domains becomes more exposed to the solvent in the $\mathrm{Zn}^{2+}$-bound state (Fig. 4b, lower and Supplementary Fig. 10B). This hydrophobic patch is not involved in the dimer interface, which accounts for the enhanced ANS fluorescence after $\mathrm{Zn}^{2+}$ addition (Fig. 1d). These structural insights suggest that the $\mathrm{Zn}^{2+}$-bound form of ERp44 recognizes its client proteins with higher affinity than the metal-free form.

$\mathrm{Zn}^{2+}$ enhances the interactions between ERp44 monomers and Erola. To investigate how $\mathrm{Zn}^{2+}$ affects ERp44-client interactions, we performed SEC and ITC analyses for the ERp44-Erola association in the presence or absence of the metal ion. To this end, we first added Erola to the $\mathrm{Zn}^{2+}$-bound ERp44 homodimers 
a
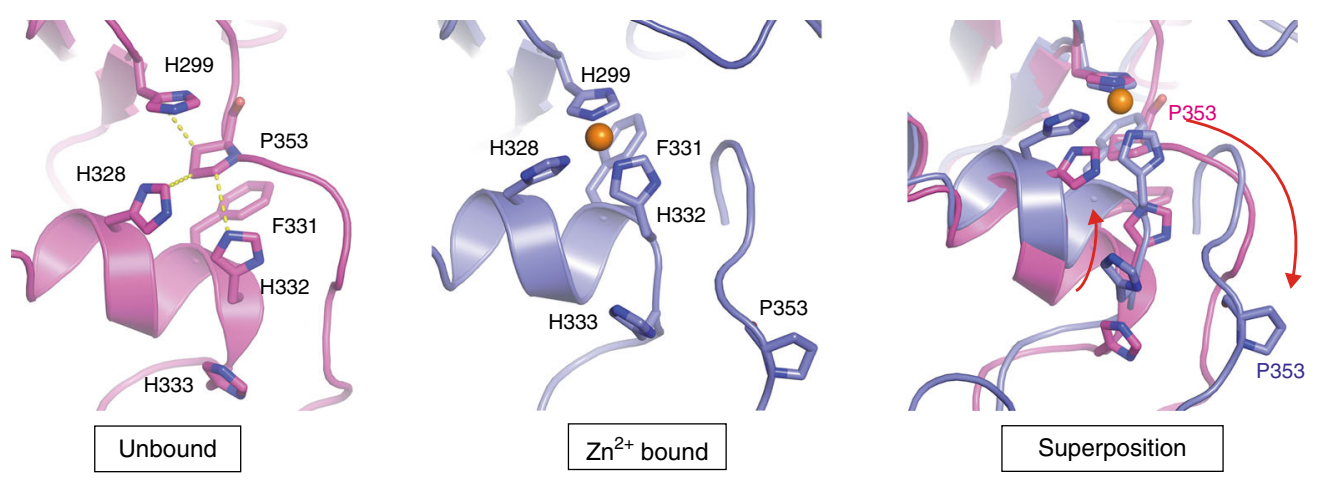

b
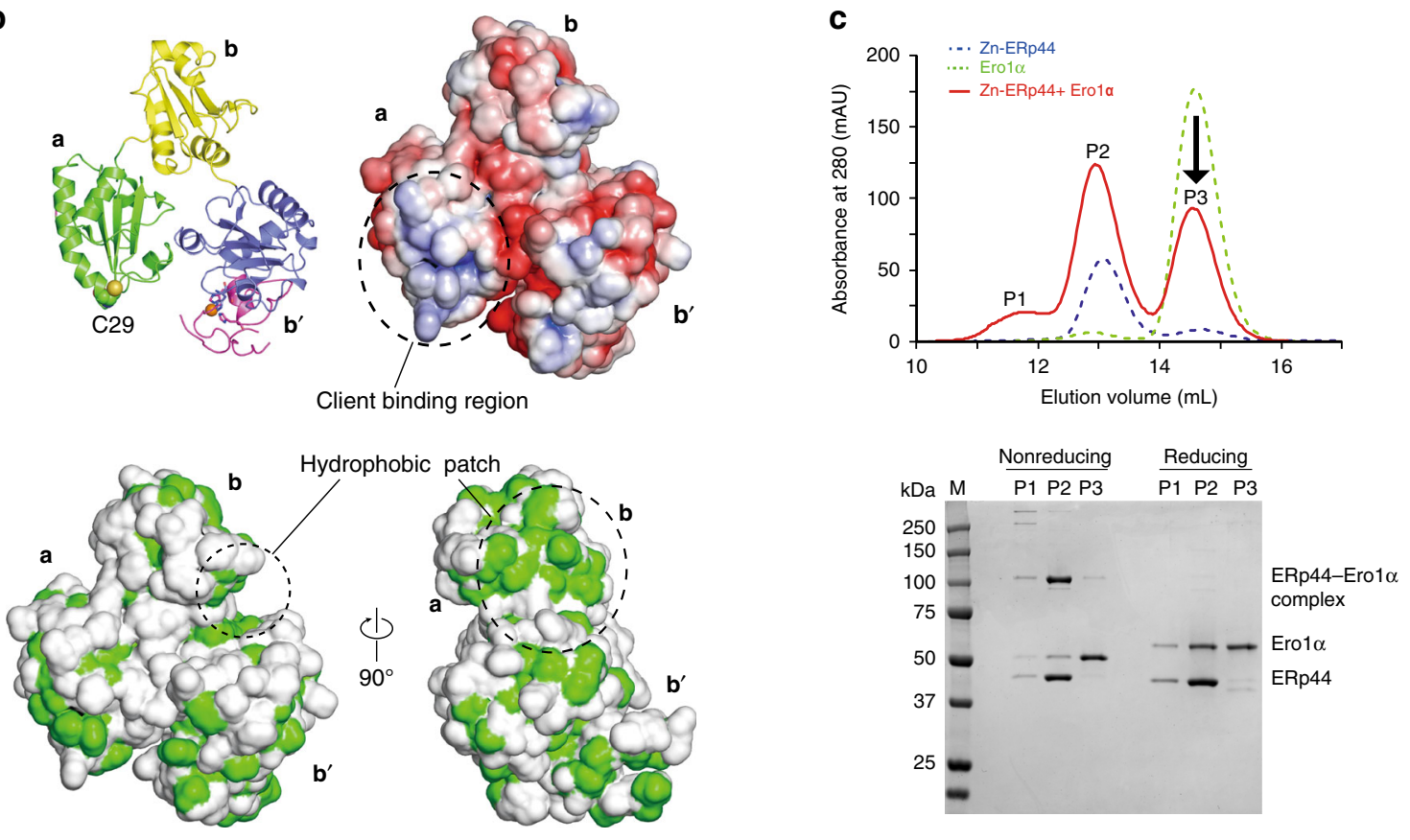

d

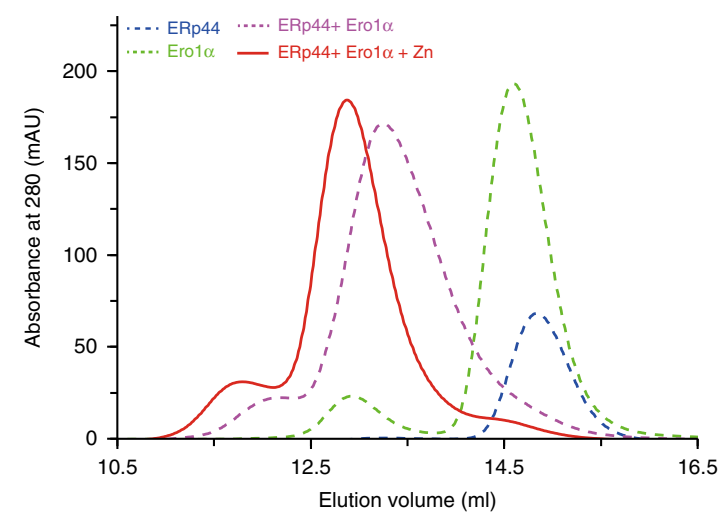

and subsequently applied the mixture to a SEC column. Clearly, whereas a minor portion of the proteins formed higher disulfidelinked oligomers (P1 fraction), a major portion of Erola coeluted with ERp44 as both disulfide-linked and non-covalent complexes (P2 fraction in Fig. 4c). The P2 fraction also contained the remainder of ERp44. The molecular mass of the P2 fraction
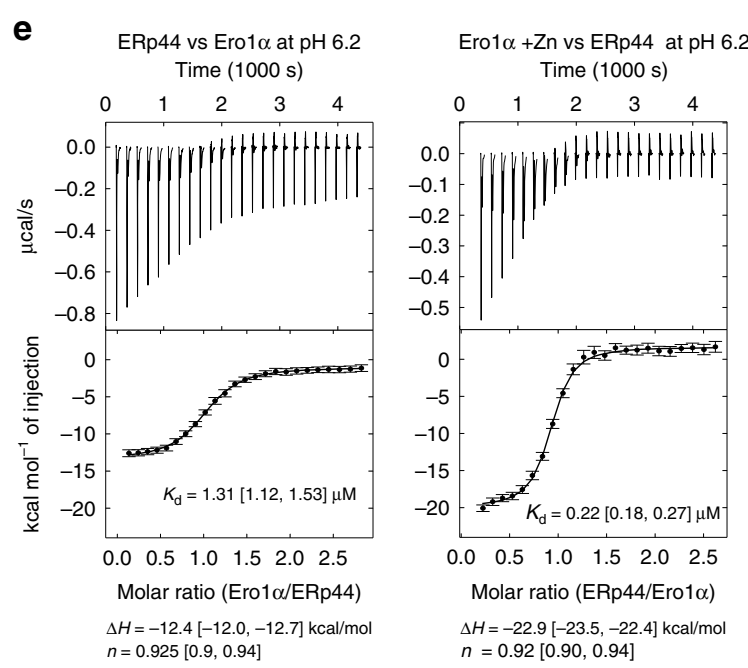

was estimated to be $\sim 90 \mathrm{kDa}$ by SEC-MALS analysis (Supplementary Fig. 11), suggesting that this fraction contains both ERp44-Erola heterodimers and $\mathrm{Zn}^{2+}$-mediated ERp44 homodimers. Thus, in the presence of Erola, significant portion of the $\mathrm{Zn}^{2+}$-bound ERp44 homodimer dissociates to form the ERp44-Ero1a complexes. 
Fig. $4 \mathrm{Zn}^{2+}$-dependent conformational changes enhance the ERp44-Ero1 $\alpha$ interaction. a Comparison of the His-cluster (site 1) conformation in the

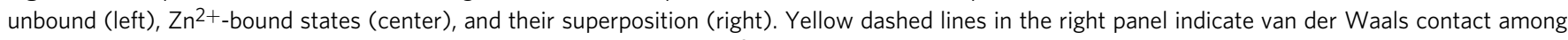
the three histidine and Pro353. b Molecular surface properties of the $\mathrm{Zn}^{2+}$-bound ERp44. (Upper left) Ribbon representation as a reference; (upper right) Electrostatic surface potential, in which positively and negatively charged regions are shown in blue and red, respectively. (Lower) Hydrophobic regions viewed from two different angles, in which hydrophobic residues (except main-chain oxygen and nitrogen atoms) are shown in green. c SEC analysis for the mixture of ERp44 (40 $\mu \mathrm{M})$ and $\mathrm{ZnCl}_{2}(80 \mu \mathrm{M})$ (dashed blue line), Ero1 $\alpha(40 \mu \mathrm{M})$ alone (dashed green line), and the pre-mixture of ERp44 (40 $\left.\mu \mathrm{M}\right)$ and $\mathrm{ZnCl}_{2}(80 \mu \mathrm{M})$ supplemented with Erol $\alpha(40 \mu \mathrm{M})$ (red line) at $\mathrm{pH}$ 6.2. Peak fractions were analyzed by SDS-PAGE under non-reducing or reducing conditions. d SEC analysis of ERp44 (40 $\mu \mathrm{M})$, Ero1 $\alpha(40 \mu \mathrm{M})$, and equimolar mixtures of ERp44 and Ero1 $\alpha(40 \mu \mathrm{M}$ each) with or without Zn $2+$ ions $(80 \mu \mathrm{M})$ at $\mathrm{pH}$ 6.2. e ITC raw data (upper) and binding isotherm data (lower) for titration of Erol $\alpha$ into $\mathrm{ERp} 44$ at $\mathrm{pH} 6.2$ (left) or titration of ERp44 into a mixture of Erol $\alpha$ and $\mathrm{Zn}^{2}+$ at $\mathrm{pH} 6.2$ (right). The calculated $K_{\mathrm{d}}$ is shown with $68.3 \%$ confidence interval in brackets

When a pre-mixture of Erola $(40 \mu \mathrm{M})$ and $\mathrm{Zn}^{2+}(80 \mu \mathrm{M})$ was incubated with ERp44 $(40 \mu \mathrm{M})$ and then applied to the column, the ERp44-Erola binary complex was formed predominantly (Fig. 4d, red line), indicating that $\mathrm{Zn}^{2+}$-bound ERp44 monomers preferentially bind Erola rather than forming $\mathrm{Zn}^{2+}$-bridged homodimers. In the presence of $\mathrm{Zn}^{2+}$, the binary complex eluted as a narrower peak with a slightly smaller elution volume (Fig. $4 \mathrm{~d}$, red and purple broken lines), suggesting that the interactions between ERp44 and Erola are enhanced by $\mathrm{Zn}^{2+}$. In support of this, the ITC analysis showed that $\mathrm{Zn}^{2+}$-dependent formation of the Erola-ERp44 complex produced larger enthalpy changes with sixfold higher affinity $\left(K_{\mathrm{d}}=0.22 \mu \mathrm{M}\right)$, compared to the $\mathrm{Zn}^{2+}$-independent association $\left(K_{\mathrm{d}}=1.31 \mu \mathrm{M}\right)$ (Fig. $\left.4 \mathrm{e}\right)$. Accordingly, TPEN treatment significantly reduced the interactions between exogenously expressed Erola-Myc and YFP-ERp44 in cells, and the subsequent ZPT treatment restored their complex formation (Supplementary Fig. 12A). Endogenous ERp44 also formed disulfide-linked binary and higher molecular weight complexes with Erola in cells, which were resolved by TPEN treatment (Supplementary Fig. 12B). Thus, $\mathrm{Zn}^{2+}$ significantly enhances the interaction between ERp44 and Erola.

To test whether ERp44 homodimerization competes with client binding also in living cells, we monitored the association of FLAG-ERp44 and YFP-ERp44 in HeLa transfectants expressing increasing amounts of Erol $\alpha$, with or without ZPT supplementation. The amount of FLAG-ERp44 co-precipitated with YFPERp44 was significantly decreased as the levels of Erola increased (Supplementary Fig. 12C). The effect of Erola co-expression was more striking when the cells were treated with ZPT. Concomitantly, ERp44-Erola complexes were generated to a greater extent. Altogether, the data support that-both in vitro and in vivo-ERp44 binds Erola with higher affinity than another ERp44 molecule and that $\mathrm{Zn}^{2+}$ further promotes the association between ERp44 and Erola.

Occurrence of $\mathrm{Zn}^{2+}$-mediated ERp44 homodimers in cells. The formation of $\mathrm{Zn}^{2+}$-dependent ERp44 homodimers was further explored by exploiting HepG2 cells that express Halo-tagged ERp44 variants (Supplementary Fig. 13A). Lysates were precipitated with Halo ligand-beads, which covalently bind the Halo domain of the chimeric proteins. After careful washing, beads were sequentially eluted with TPEN and then with DTT, to dissect non-covalent $/ \mathrm{Zn}^{2+}$-dependent and covalent interactions. TPEN treatments eluted both Halo-tagged and endogenous ERp44 molecules from beads covered with Halo-ERp44 WT, but not from beads covered with Halo-ERp44 3HA (Supplementary Fig. 13B). Similarly, when HepG2 cells coexpressed different tagged versions of WT ERp44 (Halo or HA), both were eluted from the beads by TPEN (Supplementary Fig. 13C). The association between HA-ERp44 WT and Halo-ERp44 WT was further enhanced by addition of $\mathrm{ZnCl}_{2}$ to cell lysates. (Supplementary Fig. 13C, lanes 8 and 17). Thus, $\mathrm{Zn}^{2+}$ binding is necessary to form and stabilize non-covalent homodimers also in vivo. In addition, a fraction of ERp44 WT and 3HA that formed covalent oligomers $^{27,50}$ were eluted by treating the beads with DTT (Supplementary Fig. 13A, lanes 14 and 15). Altogether, the results indicate that ERp44 WT, but not 3HA, forms $\mathrm{Zn}^{2+}$-mediated non-covalent dimers (or higher oligomers) in cells.

The occurrence of the $\mathrm{Zn}^{2+}$-bridged ERp44 homodimers including endogenous ERp44 is further supported by SEC analyses of the microsomal fraction (Supplementary Fig. 13D). In addition to monomer fractions (eluted at $\sim 14.5 \mathrm{~mL}$ ), a significant portion of endogenous ERp44 was eluted at $\sim 12.5$ $\mathrm{mL}$ (Supplementary Fig. 13E, left). Of note, the elution peak at $\sim 12.5 \mathrm{~mL}$ corresponds to that of the $\mathrm{Zn}^{2+}$-bridged ERp44 homodimers (Fig. 1c) and diminished upon TPEN treatment, but not upon tris(2-carboxyethyl)phosphine (TCEP) treatment (Supplementary Fig. 13E, middle and right). Meanwhile, TCEP markedly reduced most of the covalent complexes including ERp44 seen at $12.0-13.5 \mathrm{~mL}$. Although we cannot exclude the possibility that the earlier peak at $\sim 12.5 \mathrm{~mL}$ contains some ERp44 molecules non-covalently bound to client proteins of a molecular size similar to ERp44, these SEC analyses, taken together with the sequential elution assays shown above, indicate that ERp44 can certainly form $\mathrm{Zn}^{2+}$-dependent non-covalent and covalent homodimers in cells.

$\mathrm{Zn}^{2+}$-regulated subcellular localization and function of ERp44. To clarify the correlation between the $\mathrm{Zn}^{2+}$-binding ability and subcellular localization of ERp44, we performed cell imaging analyses for the YFP-ERp44 H277/281A (YFP-H277/281A) and $3 \mathrm{HA}$ (YFP-3HA) mutants. Due to the lack of site 1, 3HA mutant is predicted not to readily release the $\mathrm{C}$-tail in the presence of $\mathrm{Zn}^{2+}$, leading to defective recognition of the C-terminal RDEL sequence by KDELRs and hence more distal localization with respect to WT (and likely partial secretion). In agreement with our previous results ${ }^{35}$, disruption of the His-cluster (site 1) led to extensive co-localization of the mutant YFP-3HA with the cisGolgi marker GM130 (Fig. 5a, b), mimicking the phenotype observed with ERp44 WT in TPEN-treated cells (Fig. 1e and Supplementary Fig. 3). Moreover, as predicted for ERp44 mutants incapable of binding $\mathrm{Zn}^{2+}, \mathrm{ZPT}$ treatment did not affect the localization pattern of YFP-3HA (Fig. 5a, b). In line with these findings, the cells expressing YFP-3HA secreted a much larger amount of Erol $\alpha$-Myc than those expressing YFP-ERp44 WT (Fig. $5 c, d$ ). These results suggest that $\mathrm{Zn}^{2+}$ binding to the Hiscluster (site 1) is critical for both recognition by KDELRs and efficient retrograde transport of client proteins.

In sharp contrast to YFP-3HA, YFP-H277/281A, which is able to bind equimolar $\mathrm{Zn}^{2+}$ at site 1 but unable to dimerize due to the lack of site 2, preferentially accumulated in the ER. Due to its inability to form $\mathrm{Zn}^{2+}$-bridged dimers, the $\mathrm{Zn}^{2+}$-bound form of YFP-H277/281A is predicted to expose its C-terminal RDEL motif to the solvent constitutively, without concealing it inside 
a
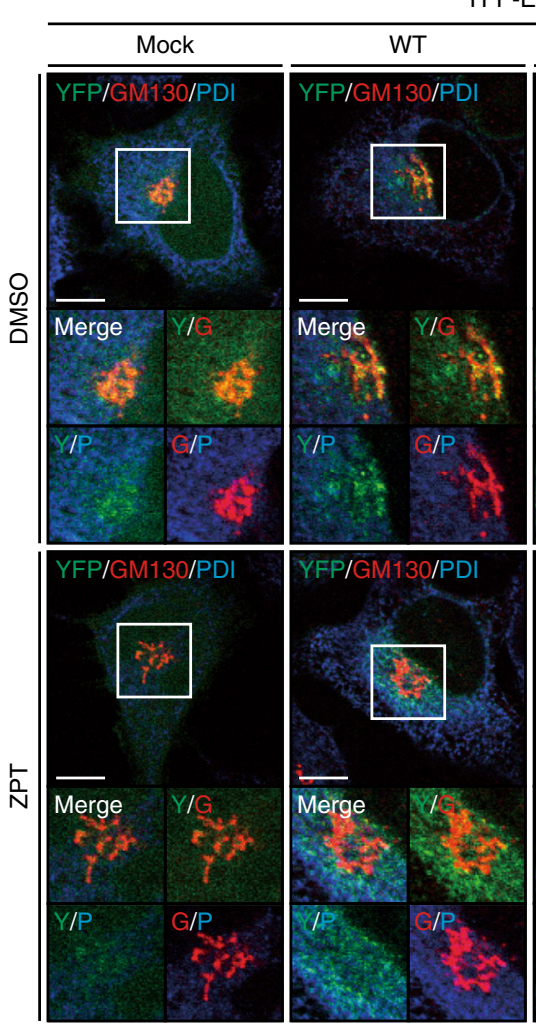

YFP-ERp44

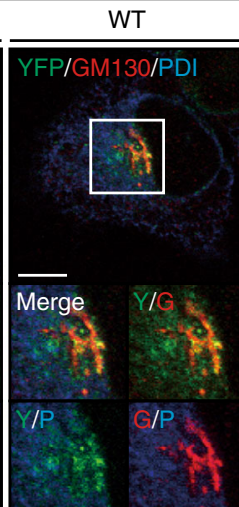

\section{.}
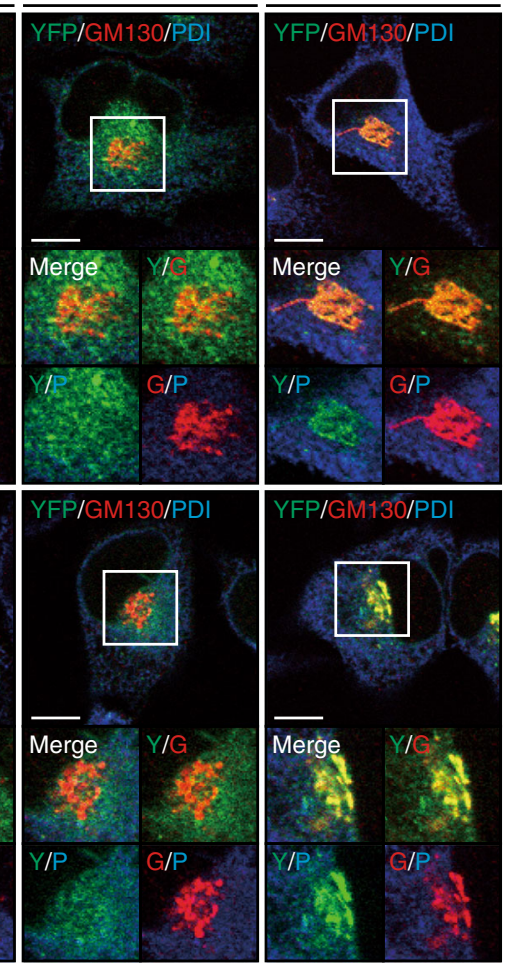

C

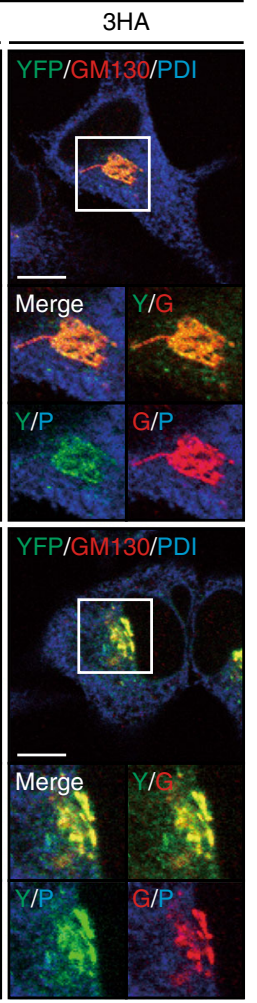

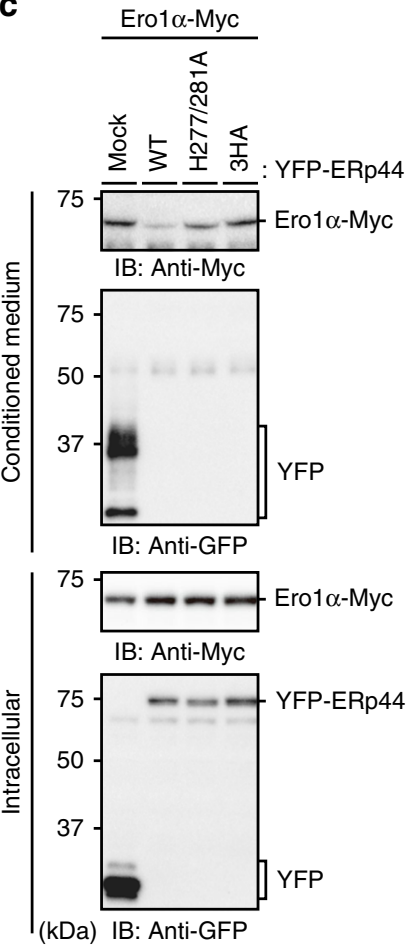

b

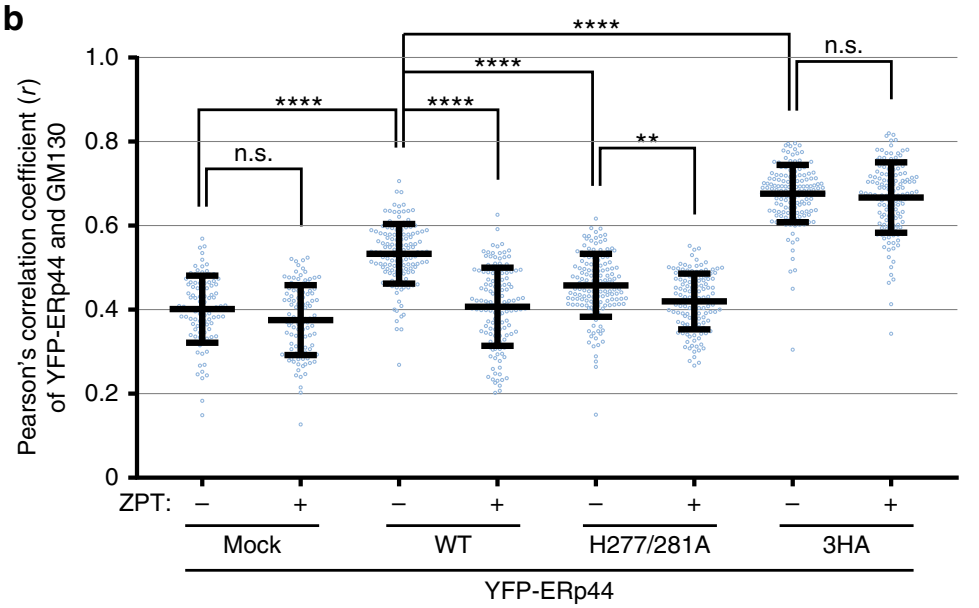

d

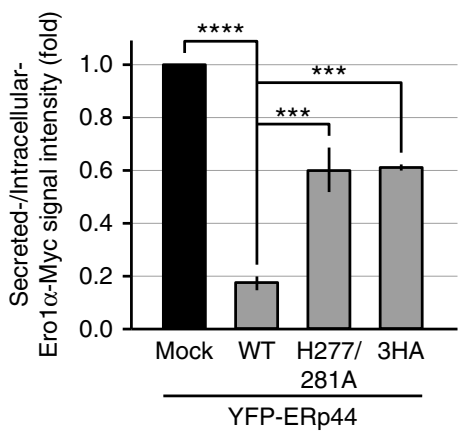

Fig. 5 Client-retention activity of ERp44 is modulated by its subcellular localization. a Confocal immunofluorescence images showing the subcellular localization of YFP-ERp44 WT, H277/281A, and 3HA in HeLa cells. HeLa cells transfected with YFP-Mock or YFP-ERp44 (WT, H277/281A, or 3HA) were treated with DMSO or $2.5 \mu \mathrm{M}$ ZPT for 15 min and immunostained for GM130 (cis-Golgi marker, in red) and PDI (ER marker, in blue). Note that YFP-ERp44 $(\mathrm{H} 277 / 281 \mathrm{~A})$ and $(3 \mathrm{HA})$ preferentially localized to the ER and Golgi, respectively. Scale bars, $10 \mu \mathrm{m}$; insets, magnification. b Quantitative analyses of Pearson's correlation coefficients of co-localization of YFP-signals with GM130 staining. Dots indicate individual data points ( $>100$ cells from three independent experiments, one-way ANOVA followed by Tukey's test). Bars indicate the means \pm SD. ${ }^{\star \star \star \star} p<0.0001 ;{ }^{\star \star} p<0.01$; n.s. not significant ( $p>$ 0.05). c, d Mutations on histidines involved in Zn ${ }^{2+}$-binding inhibit Ero1 $\alpha$ retention activity of ERp44. HeLa cells transfected with Ero1 $\alpha$-Myc and YFP-Mock or YFP-ERp44 (WT, H277/281A, or 3HA) were incubated in serum-free Opti-MEM for $6 \mathrm{~h}$. Conditioned media were precipitated with TCA and analyzed by immunoblotting with anti-GFP or anti-Myc antibodies, as indicated, under reducing conditions. The signal intensity of secreted Erol $\alpha-M y c$ relative to that of intracellular Ero1 $\alpha$-Myc was quantified, and the data are shown in the graph (d). Data are the means \pm SEM ( $N=3$, one-way ANOVA followed by Dunnett's test). ${ }^{\star \star \star \star} p<0.0001 ;{ }^{* \star *} p<0.001$

the dimer interface. However, this mutant accumulated in the Golgi upon TPEN treatment, as observed with YFP-WT (Supplementary Fig. 14), suggesting that it can potentially be exported from the ER to the post-ER compartments. Though still exhibiting $\mathrm{Zn}^{2+}$-dependent subcellular localization changes, H277/281 A was less efficient in retaining Ero1a-Myc than WT (Fig. 5c, d). These results suggest that proper cycling of ERp44 in post-ER stations is crucial for efficient Erola retention. In 
agreement with this notion, we previously reported that ERp44 mutants of which the $\mathrm{C}$-tail is constitutively open reside predominantly in the ER and retain poorly their clients ${ }^{20,32}$.

\section{Discussion}

The present studies reveal that $\mathrm{Zn}^{2+}$ regulates the structure, function and subcellular localization of ERp44, providing a $\mathrm{Zn}^{2}$ ${ }^{+}$-dependent mechanism regulating protein quality control in the ESP. $\mathrm{Zn}^{2+}$ binding to the His-cluster (site 1) induces striking structural rearrangement in ERp44, increasing sixfold its affinity for the substrate, Erola (Fig. 4e). Upon the $\mathrm{Zn}^{2+}$-induced release of the C-tail, the C-terminal RDEL motif is fully exposed to the solvent. The motif can hence be readily recognized by KDELR at the Golgi, and ERp44 is transported to the ER with high efficiency. Thus, excess $\mathrm{Zn}^{2+}$ localizes ERp44 predominantly in the ER. By contrast, $\mathrm{Zn}^{2+}$ chelation prevents the exposure of the RDEL motif by keeping the C-tail closed. ERp44 in this conformation will hardly be recognized by KDELR. As a consequence, ERp44 accumulates in the Golgi and is in part secreted. The observation that knockdown of the Golgi-resident $\mathrm{Zn}^{2+}$ transporters (ZnT5, ZnT6, and ZnT7) altered the ERp44 localization and compromised its client retention activity highlights the essential role of $\mathrm{Zn}^{2+}$ in the physiological regulation of ERp44. Like metallothioneins, ERp44 condenses diverse $\mathrm{Zn}^{2+}$-binding modes with different affinities and functional roles in a single protein. Although catalytic or structural $\mathrm{Zn}^{2+}$ binding sites in $\mathrm{Zn}^{2+}$ metalloproteins have picomolar to nanomolar affinity for $\mathrm{Zn}^{2+5}$, ERp44 binds $\mathrm{Zn}^{2+}$ with submicromolar affinity. Thus, $\mathrm{Zn}^{2+}$ likely has regulatory rather than structural roles for ERp44.

ERp44 has been found to possess three $\mathrm{Zn}^{2+}$-binding sites (sites 1, 2, and 3). Slightly lower $\mathrm{Zn}^{2+}$-binding affinity at lower $\mathrm{pH}$ can be explained by higher-degree protonation of the histidines at lower $\mathrm{pH}$. Importantly, positive cooperativity exists in $\mathrm{Zn}^{2+}$-binding to sites 1 and 2 (Fig. $4 \mathrm{a}, \mathrm{b}$ and Supplementary Fig. 6A); $\mathrm{Zn}^{2+}$ first binds to the conserved His-cluster (site 1 ), inducing large conformational changes, followed by facilitated $\mathrm{Zn}^{2+}$ binding to site 2 to form $\mathrm{Zn}^{2+}$-mediated homodimers. In line with this, superposition of metal-free ERp44 onto one protomer in the $\mathrm{Zn}^{2+}$-bound homodimer shows that the a domain in the metal-free state undergoes steric hindrance with the C-tail of another protomer in the $\mathrm{Zn}^{2+}$-bound state (Supplementary Fig. 15). To avoid this steric hindrance, significant domain rearrangements are induced by $\mathrm{Zn}^{2+}$ binding, leading to $\mathrm{Zn}^{2+}$-bridged dimerization. Site 3 has much lower $\mathrm{Zn}^{2+}$ affinity than the other two sites (Fig. 1b, c and Supplementary Fig. 8C). Nonetheless, mutating site-3 histidine (His333) weakened the Erola retention activity of ERp $44^{37}$. The His333 mutation likely disrupts the dimer interface (Fig. 3b, right, Supplementary Fig. 7), thereby influencing the configurations of sites 1 and 2 and compromising the overall responsiveness of ERp44 to $\mathrm{Zn}^{2+}$. In the ESP, the potential binding of five $\mathrm{Zn}^{2+}$ atoms may enable ERp44 homodimers to function as $\mathrm{Zn}^{2+}$ buffer/storage devices, in analogy to cytoplasmic metallothioneins that bind up to seven metal ions ${ }^{12,51}$.

The present findings allow us to propose a model of $\mathrm{Zn}^{2+}$-dependent protein quality control in the ESP (Fig. 6). At low $\mathrm{Zn}^{2+}$ concentration and neutral $\mathrm{pH}$ as in the ER, ERp44 likely assumes a closed conformation, with its client-binding surface masked by the C-tail ${ }^{32,34}$. At the weakly acidic $\mathrm{pH}$ of the cis-Golgi, ERp44 undergoes pH-dependent conformational changes that increase the $\mathrm{C}$-tail dynamics and Cys29 reactivity and expose positively charged regions around the client-binding site ${ }^{32,34} . \mathrm{Zn}^{2+}$ binding further releases the C-tail, enhancing the interactions with clients and KDELRs. In the absence of clients,
ERp44 is likely in equilibrium between a metal-free monomer, a $\mathrm{Zn}^{2+}$-bound monomer and a $\mathrm{Zn}^{2+}$-bridged homodimer in postER compartments (Fig. 6). Once the clients arrive at the Golgi, the $\mathrm{Zn}^{2+}$-bound ERp44 homodimers can dissociate to monomers to form binary complexes with clients. The ERp44-client complex is in turn retrieved to the ER in concert with KDELRs. In this scenario, the $\mathrm{Zn}^{2+}$-bridged ERp44 homodimer can be regarded as reservoirs that are ready to tightly bind clients. The dimer may also serve to store the metal in the ESP under $\mathrm{Zn}^{2+}$-limiting conditions.

How does $\mathrm{Zn}^{2+}$ participate in the ERp44 cycle under physiological settings? In mammalian cells, the total $\mathrm{Zn}^{2+}$ concentration including the protein-bound fraction is estimated to be $100-500$ $\mu \mathrm{M}^{15,52}$. Recent $\mathrm{X}$-ray fluorescence microscopy studies of single cells have shown that $\mathrm{Zn}^{2+}$ is transiently stored in the Golgi ${ }^{53,54}$, where the metal is imported by ZnT5, ZnT6, and ZnT7. Notably, $\mathrm{ZnT}$ s are proposed to act as $\mathrm{Zn}^{2+} / \mathrm{H}^{+}$antiporters ${ }^{10}$. Thus, $\mathrm{Zn}^{2+}$ uptake into the Golgi is most likely coupled with $\mathrm{H}^{+}$efflux into the cytosol. Accordingly, $\mathrm{Zn}^{2+}$ import activity is enhanced by Golgi acidification ${ }^{55}$, highlighting the crosstalk between $\mathrm{pH}$ and $\mathrm{Zn}^{2+}$ in the ESP. Conversely, the neutral $\mathrm{pH}^{32}$ and the paucity of $\mathrm{ZnTs}^{11}$ in the ER likely induce ERp44 to adopt the closed conformation, allowing client release. Previous studies reported that free $\mathrm{Zn}^{2+}$ concentration in the ER is kept within the range of $\sim 1$ $\mathrm{pM}$ or $1-5 \mathrm{nM}$ in mammalian cells ${ }^{56-59}$. Such low $\mathrm{Zn}^{2+}$ concentrations and the neutral $\mathrm{pH}$ may synergize in facilitating client dissociation possibly with the help of unidentified reductases or other factors. Altogether, we propose that the $\mathrm{pH}$ - and the $\mathrm{Zn}^{2}$ ${ }^{+}$-gradients in the ESP collaboratively modulate the structure and function of ERp44, enabling cycles of client binding, retrieval and release.

In this context, it is noteworthy that the expression of $\mathrm{Zn}^{2+}$ transporters (ZnT6, ZnT7, ZIP7, ZIP9, and ZIP13) in the Golgi is upregulated in conditions of $\mathrm{Zn}^{2+}$ deficiency or ER stress ${ }^{60}$. ER stress is also known to induce the expression of $\mathrm{ZnT}^{61}$ as well as of ERp44 and ERGIC-53 ${ }^{50,62}$. The co-regulation of ERp44 and $\mathrm{ZnTs} / \mathrm{ZIPs}$ under stress conditions supports our notion that ERp44 is a $\mathrm{Zn}^{2+}$-dependent ESP chaperone.

Our findings suggest that the $\mathrm{Zn}^{2+}$-dependent subcellular localization of ERp44 is key to efficient client retention. Unlike PDI and other ER chaperones with KDEL-like motifs ${ }^{63,64}$, endogenous ERp44 localizes in ERGIC and cis-Golgi at steady state and is readily secreted upon RDEL deletion ${ }^{27,28}$. Thus, ERp44 rapidly cycles throughout the ESP. Mutations at site 1 or at site 2 lead to its predominant accumulation in the Golgi or the $\mathrm{ER}$, respectively. Importantly, both mutations compromised ERp44 activity. We surmise that the rapid arrival of ERp44 in the ERGIC and cis-Golgi is beneficial to control the integrity of the secretome by surveying and capturing assembly intermediates or ER residents that gain access to those compartments. Rapid cycling-possibly involving ERGIC-53 $3^{28}$-may enable sufficient ERp44 to accumulate in post-ER compartments in a conformation ready to bind clients and retrieve them to the $\mathrm{ER}^{32}$.

In conclusion, the metal-dependent allosteric activation of ERp44 reveals a regulatory role of $\mathrm{Zn}^{2+}$ for protein quality control in the ESP, besides its well-known functions in the metalloenzymes biogenesis ${ }^{11}$ and in transcriptional regulation ${ }^{5,65}$. Metal response element-binding transcription factor-1 senses cellular free $\mathrm{Zn}^{2+}$ through six zinc-finger motifs, controlling the expression of $\mathrm{Zn}^{2+}$ exporters and $\mathrm{Zn}^{2+}$ storage proteins, metallothioneins ${ }^{66}$. Transient $\mathrm{Zn}^{2+}$ binding is also known to inhibit several enzymes and transporters ${ }^{67-69}$. Thus, $\mathrm{Zn}^{2+}$ signaling is involved in a variety of regulatory pathways. Our work sheds light on yet another aspect of $\mathrm{Zn}^{2+}$ biology, with potential implications in surveilling the metallo-secretome and maintaining $\mathrm{Zn}^{2+}$ homeostasis. 


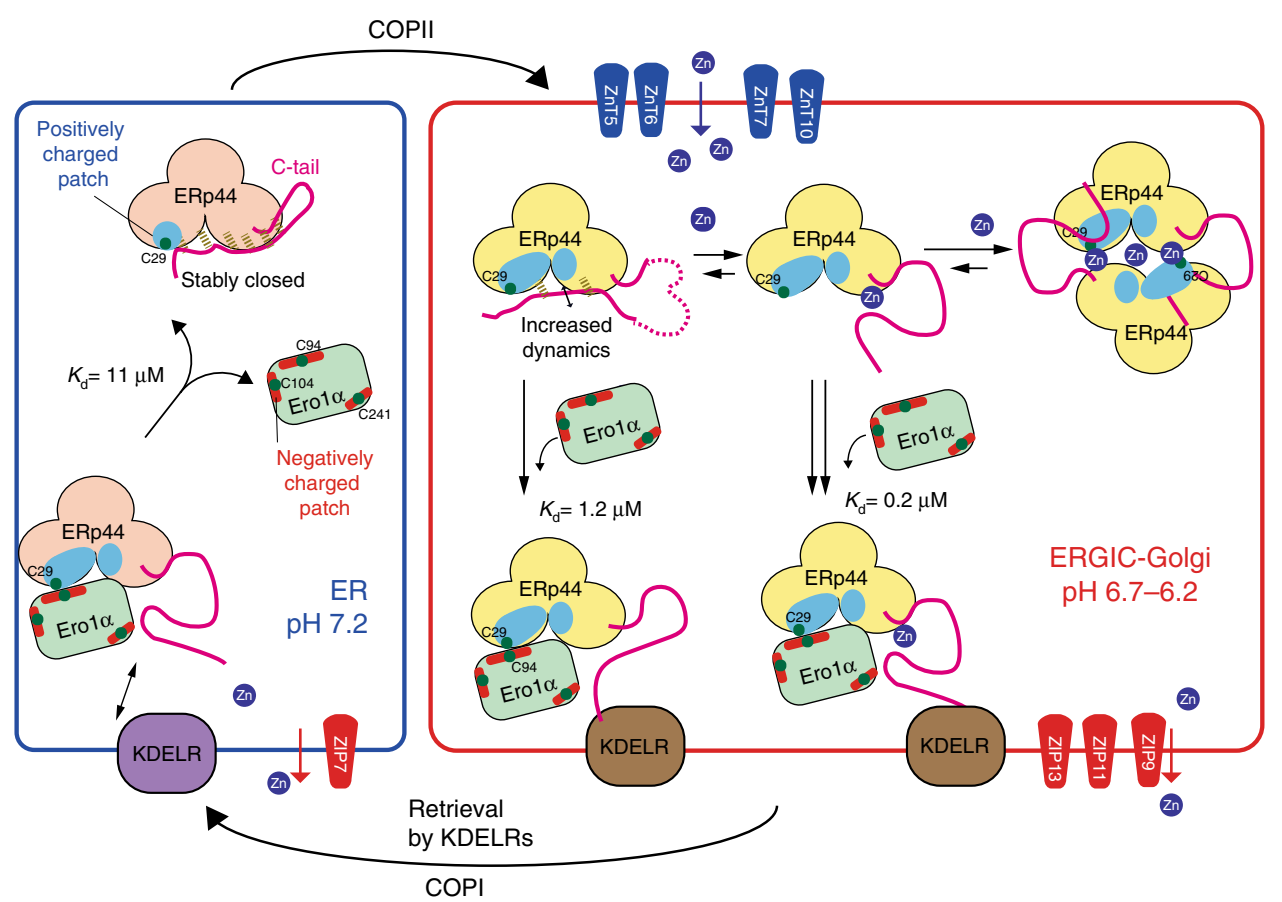

Fig. $6 \mathrm{Zn}^{2+}$-dependent and pH-dependent regulation of ERp44 for efficient client retention in the early secretory pathway. Proposed working model of the $\mathrm{Zn}^{2+}$-dependent and $\mathrm{pH}$-dependent ERp44 cycle operating for protein quality control in the ESP. In the ERGIC and cis-Golgi, ERp44 is in equilibrium between monomeric metal-free, monomeric $\mathrm{Zn}^{2+}$-bound, and $\mathrm{Zn}^{2+}$-mediated homodimeric conformations. Upon the arrival of a client like Erol $\alpha$, the equilibrium is shifted in favor of the monomeric state with the C-tail opened, favoring the exposure of the RDEL motif. Thus, the ERp44-client complex is promptly bound by KDELR and therewith transported back to the ER. The lower $\mathrm{Zn}^{2+}$ concentration in the neutral ER likely promotes the dissociation of the ERp44-client complexes, allowing another ERp44 cycle through the ESP

\section{Methods}

Reagents and antibodies. 4',6-diamidino-2-phenylindol (DAPI; Polysciences, Warrington, PA), $N$-ethylmaleimide (NEM; Nacalai Tesque), $N, N, N^{\prime}, N^{\prime}$-Tetrakis (2-pyridylmethyl)ethylenediamine (TPEN; Dojindo Laboratories), Tetraethylenepentamine (TEPA; Sigma), and Zinc pyrithione (ZPT; Tokyo Chemical Industry) were commercially purchased. Antibodies were purchased as follows: mouse monoclonal antibodies against c-Myc (9E10; Santa Cruz) (1:10,000 for western blot (WB)), green fluorescent protein (GFP; 7.1 and 13.1; Roche) (1:1000 for WB), FLAG (M2; Sigma) (1:10,000 for WB), HA (12CA5; purified from 12CA5 hybridoma supernatant (ATCC)) (1:1000 for WB) and PDI (1D3; Enzo Life Sciences) (1:1000 for both WB and immunofluorescence (IF)), rabbit monoclonal antibodies against ERp44 (D17A6; Cell Signaling Technology) (1:1000 for WB), Rab7 (D95F2; Cell Signaling Technology) (1:200 for IF), and rabbit polyclonal antibodies against Calnexin (ADI-SPA-860; Enzo Life Sciences) (1:200 for IF), EEA1 (PM062; MBL) (1:200 for IF), ERGIC-53 (E1031; Sigma) (1:2000 for IF), GFP (A-6455; Molecular Probes) (1:5000 for WB), Halo (G9281; Promega) (1:1000 for WB) and GM130 (PM061; MBL; and a kind gift of Dr. A. De Matteis, Naples) (1:1000 for IF). The anti-Erola 2G4 monoclonal antibody was raised using the chimera GST-Erol $\alpha$ as an antigen (ARETA International S.r.l.), as described previously ${ }^{70}$, and its specificity was validated by several assays, including ELISA, IF and immunoprecipitation. 2G4 antibodies were used at a 1:1000 dilution for WB. Polyclonal antibodies against human PDI (A66) were described previously ${ }^{71}$ and used at 1:500 for WB. Monoclonal anti-ERp44 antibodies (clones 36C9 and 2D5) (36C9; 1:1000 for WB, 2D5; 1:50 for IF) were previously described ${ }^{28,50} . \mathrm{Zn}^{2+}$ solution used for the experiments was prepared by diluting a stock solution of $2 \mathrm{M}$ $\mathrm{ZnCl}_{2}$ in $\sim 20 \mathrm{mM} \mathrm{HCl}$ with the indicated buffer. The stock solution and titrant solution were prepared just before measurements.

Purification and characterization of ERp44 and Ero1 $\alpha$. A series of His to Ala mutations were introduced into bacterial expression vectors using a quick-change mutagenesis kit (Agilent) or a PCR-based method with appropriate sets of primers (Takara) (Supplementary Table 4). Designated mutations were verified by sequencing. Overexpression and purification of recombinant WT or mutant ERp44 variants (Supplementary Table 4) were performed as described previously ${ }^{32}$. Erola Cys104/Cys131Cys166Ala mutant was prepared as described previously ${ }^{39}$ and used in all the experiments. The histidine tag of all recombinant samples was cleaved by thrombin treatment.

ANS fluorescence spectra were recorded in $1 \mathrm{~cm}$ cuvettes on a Hitachi F-7000 spectrofluorometer as described previously ${ }^{37}$. Samples were prepared as follows: (a) recombinant ERp44 WT ( $5 \mu \mathrm{M})$ in buffer ( $20 \mathrm{mM}$ BisTris-HCl pH 7.2 or 6.2 , $150 \mathrm{mM} \mathrm{NaCl})$; (b) ERp44 $(5 \mu \mathrm{M})$ was mixed with $10 \mu \mathrm{M} \mathrm{ZnCl} 2$ and incubated at $293 \mathrm{~K}$ for $5 \mathrm{~min}$; (c) subsequent addition of EDTA (1 mM) into sample B and further incubated at $293 \mathrm{~K}$ for $5 \mathrm{~min}$. These samples were mixed with $40 \mu \mathrm{M}$ ANS, and incubated at $293 \mathrm{~K}$ for $10 \mathrm{~min}$ before measurement.

The far-UV CD spectra were recorded with ERp44 WT and its mutants $(5.7 \mu \mathrm{M})$ in $10 \mathrm{mM}$ Bis-Tris $\mathrm{pH} 7.0$ on a J-720 CD spectropolarimeter (JASCO) at room temperature using a cuvette of $1 \mathrm{~mm}$ path-length.

Isothermal titration calorimetry analysis. Isothermal titration calorimetry (ITC) experiments were carried out using the MicroCal ${ }^{\mathrm{mw}}$ iTC200 calorimeter (GE Healthcare). Concentrated samples were diluted into $20 \mathrm{mM}$ BisTris- $\mathrm{HCl} \mathrm{pH} \mathrm{6.2,}$ 6.7 , or 7.2 and $150 \mathrm{mM} \mathrm{NaCl}$ at $283 \mathrm{~K}$. Typically, after a $0.4 \mu \mathrm{L}$ initial injection, 0.8 or $1 \mu \mathrm{L}$ of titrant solution was injected into protein solution at $150 \mathrm{~s}$ intervals with stirring $750 \mathrm{rpm}$. For $\mathrm{Zn}^{2+}$ binding analysis, 48 injections of $0.8 \mu \mathrm{L}$ of $500 \mu \mathrm{M}$ $\mathrm{ZnCl}_{2}$ were added into $30 \mu \mathrm{M}$ ERp44. For the interaction between ERp44 and Erola at pH 6.2, 24 injections of $1.5 \mu \mathrm{L}$ of $420 \mu \mathrm{M}$ Erola were added into $30 \mu \mathrm{M}$ ERp44 WT. For the interaction between ERp44 and Erola in the presence of $\mathrm{Zn}^{2+}$ at $\mathrm{pH} 6.2,24$ injections of $1.5 \mu \mathrm{L}$ of $200 \mu \mathrm{M}$ ERp 44 were added into $15 \mu \mathrm{M}$ Erola and $15 \mu \mathrm{M} \mathrm{ZnCl}_{2}$. For dilution titration, 20 injections of $2 \mu \mathrm{L}$ of 10 or $100 \mu \mathrm{M}$ ERp44 with 20 or $200 \mu \mathrm{M} \mathrm{ZnCl}_{2}$ in $20 \mathrm{mM}$ BisTris pH 6.2, $150 \mathrm{mM} \mathrm{NaCl}$ were added into the same buffer. Each condition was repeated at least twice.

The integration of the observed injection peaks was performed with NITPIC ${ }^{72}$ and the global analysis was performed with SEDPHAT ${ }^{73}$. We thereby obtained stoichiometry $(n)$, apparent binding affinity $\left(K_{\mathrm{a}}\right)$ and enthalpy change $(\Delta H)$ for $\mathrm{Zn}^{2+}$ binding to ERp44 using a 1:1 binding model, as described in the previous literature ${ }^{74}$.

Scatchard plots of the observed ITC data for $\mathrm{Zn}^{2+}$-binding to ERp44 were calculated as described previously ${ }^{48}$. Briefly, the fraction of bound $\mathrm{Zn}^{2+}$ vs. total ERp44 $\left(R(i)=X_{\text {bound }}(i) / M_{\mathrm{t}}(i)\right)$ was plotted against $R(i) / X_{\text {free }}(i)$, where $X_{\text {bound }}(i)$ is the concentration of $\mathrm{Zn}^{2+}$ bound to ERp44 after the $i$-th injection, $M_{\mathrm{t}}(i)$ is the total concentration of ERp44 after the $i$-th injection, which was automatically corrected by the Origin program considering the volume increase caused by every injection, and $X_{\text {free }}(i)$ is the concentration of free $\mathrm{Zn}^{2+}$ after the $i$-th injection. The $X_{\text {bound }}(i)$ value was calculated using the following equation:

$$
X_{\text {bound }}(i)=\left[Q(i) /\left(\left(\Delta H+q_{\text {dilute }}\right) V_{0}\right)\right]+X_{\text {bound }}(i-1)
$$

where $Q(i)$ is the heat generated on the $i$-th injection measured by the ITC experiment, $\Delta H$ is the enthalpy change, $q_{\text {dilute }}$ is estimated normalized heat of 
dilution, and $V_{0}$ is the active cell volume. The $X_{\text {free }}(i)$ value was calculated using the following equation:

$$
X_{\text {free }}(i)=X_{\mathrm{t}}(i)-X_{\text {bound }}(i)
$$

where $X_{\mathrm{t}}(i)$ is the total concentration of $\mathrm{Zn}^{2+}$ after the $i$-th injection.

In the saturation plots based on the observed ITC, $X_{\text {free }}(i)$ was plotted against the fractional saturation $\left(R(i)=X_{\text {bound }}(i) / M_{\mathrm{t}}(i)\right)$. The hill coefficients were estimated with Prism software version 7.0a (GraphPad Software).

SEC and SEC-MALS analysis. SEC was performed with a superdex 200 increase $10 / 300$ column (GE healthcare) using an ÄKTA explorer $10 \mathrm{~S}$ system. $50 \mu \mathrm{L}$ of samples containing $60 \mu \mathrm{M}$ ERp44 WT or mutants and $120 \mu \mathrm{M} \mathrm{ZnCl}_{2}$ ions was incubated on ice for $>30 \mathrm{~min}$ and then loaded onto the column equilibrated in 20 mM BisTris-HCl pH 7.0 (Fig. 1c), 6.2 (Fig. 4d, Supplementary Fig. 1B), 6.7 (Supplementary Figs. 1B and 8C), or 7.2 (Supplementary Figs. 1B) and $150 \mathrm{mM}$ $\mathrm{NaCl}$ at $277 \mathrm{~K}$. Fractions were treated with $20 \mathrm{mM}$ NEM for $>10 \mathrm{~min}$ and thereafter analyzed on 10\% SDS-PAGE gels. For the complex between ERp44 and Erola in the presence of $\mathrm{Zn}^{2+}$, a mixture of $40 \mu \mathrm{M}$ Erola and $80 \mu \mathrm{M} \mathrm{ZnCl}_{2}$ was mixed with equimolar ERp44 and was incubated on ice for $>5 \mathrm{~min}$ and then loaded onto the column equilibrated in $20 \mathrm{mM}$ BisTris- $\mathrm{HCl} \mathrm{pH} 6.2$ and $150 \mathrm{mM} \mathrm{NaCl}$ at $277 \mathrm{~K}$. For the interaction between the $\mathrm{Zn}^{2+}$-bound ERp44 with Erola, a mixture of $40 \mu \mathrm{M}$ ERp44 and $80 \mu \mathrm{M} \mathrm{ZnCl}$ was mixed with $40 \mu \mathrm{M}$ Erola and was incubated on ice for $>5 \mathrm{~min}$ and then loaded onto the column.

SEC-multi-angle light scattering (MALS) experiments were carried out with a superdex 200 10/300 column equilibrated in $20 \mathrm{mM}$ BisTris pH 7.0 and $150 \mathrm{mM}$ $\mathrm{NaCl}$ using an ÄKTA FPLC system. $50 \mu \mathrm{L}$ of samples containing $60 \mu \mathrm{M}$ ERp44 WT and three fold excess $\mathrm{Zn}$ ions was incubated on ice for $>30 \mathrm{~min}$ and then loaded onto the column at room temperature. For the $\mathrm{Zn}^{2+}$-bound ERp44-Erol $\alpha$ interaction, a mixture of $60 \mu \mathrm{M}$ ERp44 and $120 \mu \mathrm{M} \mathrm{ZnCl}_{2}$ was mixed with $60 \mu \mathrm{M}$ Erola and was incubated on ice for $>30 \mathrm{~min}$ and then loaded onto the column. The column was connected to a miniDAWN TREOS detector, followed by a refractiveindex detector RI-101 (Shodex). Molecular masses were calculated with ASTRA V (Wyatt Technology) with the $\mathrm{d} n / \mathrm{d} c$ value of $0.185 \mathrm{~mL} / \mathrm{g}$.

Crystallization. Crystals of the ERp44- $\mathrm{Zn}^{2+}$ complex grew for several days in the sitting drop vapor diffusion method at $277 \mathrm{~K}$, by mixing $0.7 \mu \mathrm{L}$ of the protein solution ( $10 \mathrm{mg} / \mathrm{mL}$ protein, $0.5-2 \mathrm{mM} \mathrm{ZnCl}_{2}, 20 \mathrm{mM}$ HEPES-NaOH pH 7.0, and $150 \mathrm{mM} \mathrm{NaCl}$ ) with $0.7 \mu \mathrm{L}$ of the precipitant solution $(1.0 \mathrm{M} \mathrm{Na}$ malonate, $0.09 \mathrm{M}$ HEPES-NaOH pH 7.0, 0.45\% v/v Jaffamine $\left.{ }^{\oplus} \mathrm{ED}-2001 \mathrm{pH} 7.0\right)$ or $39 \%$ Tascimate. Before data collection, a cryoprotectant solution (2.2 M Na malonate $\mathrm{pH} 7.0,0.1 \mathrm{M}$ HEPES-NaOH pH 7.0, 0.5-2 $\mathrm{mM} \mathrm{ZnCl}_{2}$ ) was added into drops several times and then crystals were flash-cooled in a nitrogen stream at $100 \mathrm{~K}$.

Data collection and structure determination. The X-ray diffraction data were collected at a wavelength of $0.9 \AA$ on beamline BL44XU at SPring-8 (Proposal No., 2014A/B6904, 2015A/B6558, 2016A/B6656) and at a wavelength of $1.1 \AA$ on beamline BL1A at the Photon factory. The diffraction images were processed with $\mathrm{XDS}$ and XSCALE ${ }^{75}$. The structure of the ERp44- $\mathrm{Zn}^{2+}$ complex was determined at $2.45 \AA$ resolution by the SAD method using anomalous signals of $\mathrm{Zn}^{2+}$ (Supplementary Table 5). We first sought to determine the structure of $\mathrm{Zn}^{2+}$-bound ERp44 by molecular replacement using previously published crystal structures of Zn-free ERp44 (PDB ID: 2R2J, 5GU6, and 5GU7) and a variety of their fragments containing one or two thioredoxin (Trx)-like domains as search models. However, we anyhow failed to reach the correct answer. We therefore decided to employ the SAD method using anomalous signals of bound $\mathrm{Zn}^{2+}$ for phase determination. As we could not collect diffraction datasets with high redundancy from a single crystal due to radiation damage, we merged and scaled anomalously multiple isomorphous datasets with a total rotation range of $\sim 80^{\circ}$ for each (Supplementary Table 6). Merging seven datasets with high CC values allowed the SAD phasing and improved resolution and statistics compared to each native dataset. Consequently, clear electron density maps were provided for the entire regions including the Ctail. Substructure determination, phasing and density modification were performed with SHELXD and SHELXE ${ }^{76}$, followed by auto-model building with Buccaneer ${ }^{77}$. Further manual model building was performed with $\mathrm{COOT}^{78}$, refined with PHE$\mathrm{NIX}^{79}$ and validated with MolProbity ${ }^{80}$. A Fo-Fc map showed two additional positive peaks nearby $\mathrm{Zn} 3$, to which two $\mathrm{Cl}^{-}$ions were assignable, since placing $\mathrm{Cl}^{-}$ions at these positions, but not water molecules, resulted in no extra electron density in the Fo-Fc map and gave reasonable B-factors for these ions (Supplementary Table 7). Structural Figures were prepared with PyMOL (http://www. pymol.org) and Chimera ${ }^{81}$.

Cell culture and transfection. HepG2 cells (purchased from ATCC) were grown in Dulbecco's modified Eagle's medium (DMEM; Nacalai Tesque) containing 10\% fetal calf serum (FCS). Different HeLa sublines were utilized and kept at 5 or $10 \%$ FCS in DMEM. Small interfering RNAs (siRNAs) duplexes were transfected using Lipofectamine RNAiMAX reagent (Life Technologies). Plasmids were transfected using FuGENE-HD transfection reagent (Promega) (Figs. 1g, 5a, $\mathrm{c}$ and Supplementary Fig. 3B, 4C, 4H, 12A, 12C and 14) or as described previously in Vavassori et al. ${ }^{22}$ (Fig. $2 \mathrm{c}$ and Supplementary Fig. 13).
Immunofluorescence. To analyze the localization of endogenous ERp44 in HeLa cells, cells plated onto poly-L-lysine coated coverslips were incubated in Opti-MEM containing $10 \mu \mathrm{M}$ TPEN for $30 \mathrm{~min}$ and some of them were further incubated in Opti-MEM containing $20 \mu \mathrm{M} \mathrm{ZnSO}_{4}$ for $3 \mathrm{~h}$. Since chloride ions are known to precipitate $\mathrm{Fe}^{3+}$, an essential metal ion, at the neutral $\mathrm{pH}$, we used $\mathrm{ZnSO}_{4}$ instead of $\mathrm{ZnCl}_{2}$ to supplement $\mathrm{Zn}^{2+}$ to cultured cells. Cells were fixed with PBS containing 4\% PFA, permeabilized with PBS containing $0.1 \%$ TritonX-100 and blocked with PBS containing $2 \%$ FCS for $1 \mathrm{~h}$ at room temperature. Cells were then incubated with antibodies against ERp44 (2G5) and GM130 for 90 min. CF488conjugated anti-mouse IgG and CF568-conjugated anti-rabbit IgG antibodies (Biotium) were used as secondary antibodies. Nuclei were stained with DAPI. Fluorescent images were obtained using a confocal microscope (FV1000, Olympus) equipped with a UPLSAPO 60x silicon oil-immersion objective lens (NA 1.30). Pearson's correlation coefficient values were calculated using ImageJ software.

For HepG2 cells, cells plated onto $13 \mathrm{~mm}$ coverslips were treated as described above. At the end of treatments, cells were fixed with PBS containing $4 \%$ paraformaldehyde, washed and incubated with $0.1 \%$ glycine in PBS for $15 \mathrm{~min}$, permeabilized with PBS containing $0.1 \%$ TritonX-100, and blocked in PBS containing 5\% FCS and 5\% BSA for 30 min at RT. Cells were then incubated with or without monoclonal antibodies against ERp44 (2D5) for $1 \mathrm{~h}$, then with rabbit anti-GM130 antibodies for $30 \mathrm{~min}$ at RT. After extensive washing, slides were incubated with Alexa Fluor 546 goat anti-rabbit and Alexa Fluor 647 goat antimouse (Thermo Fischer Scientific) secondary antibodies. Nuclei were stained with Hoechst (1:1000). Fluorescent images were obtained using a confocal laser scanning microscopy (Leica TCS SP8 SMD FLIM) equipped with an oil lens (HC PL APO CS $2 \times 63$ (NA 1.4)) and deconvoluted with Huygens Essential. Pearson's correlation coefficient values were calculated using the Volocity software (PerkinElmer). The experiments with ZnT5/6/7 triple knockdown were processed similarly.

To analyze the intracellular localization of YFP-ERp44 and its mutants, HeLa cells plated on poly-L-lysine coated coverslips were transfected with plasmids and cultured for $36 \mathrm{~h}$. Cells were washed with $1 \times \mathrm{HHBSS}(5.36 \mathrm{mM} \mathrm{KCl}, 137 \mathrm{mM} \mathrm{NaCl}$, $16.65 \mathrm{mM}$ D-Glucose, and $30 \mathrm{mM}$ HEPES, $\mathrm{pH} 7.4)^{82}$ three times, preincubated in Opti-MEM for $30 \mathrm{~min}$, and then stimulated with TPEN $(10 \mu \mathrm{M})$ for $30 \mathrm{~min}$ and subsequent ZPT (final $20 \mu \mathrm{M}$ ) for $15 \mathrm{~min}$. DMSO was used as a vehicle control for each steps. Cells were then fixed and immunostained for GM130 and PDI as described above. CF633-labeled anti-mouse IgG, and CF568-labeled anti-rabbit IgG antibodies (Biotium) were used as the secondary antibodies. Nuclei were stained with DAPI. Fluorescent images were obtained using a confocal microscope (FV1000, Olympus) equipped with a UPLSAPO $\times 60$ oil-immersion objective lens (NA 1.35) or UPLSAPO $\times 60$ silicon oil-immersion objective lens (NA 1.30). Pearson's correlation coefficient values were calculated using ImageJ software.

Time-lapse analysis. HeLa cells $\left(1.0 \times 10^{5}\right.$ cells $)$ were plated on poly-L-lysine coated glass bottomed dish and transfected with plasmids. After incubation for $36 \mathrm{~h}$, cells were further cultured in Opti-MEM for $1 \mathrm{~h}$ and then subjected to timelapse analysis using a confocal microscope (FV1000, Olympus) equipped with a UPLSAPO $\times 60$ oil-immersion objective lens (NA 1.35). Cells were treated with TPEN (final $10 \mu \mathrm{M}$ ) at $T=2 \mathrm{~min}$ and subsequently ZPT (final $20 \mu \mathrm{M}$ ) at $T=32$ min. We used ZPT to increase intracellular $\mathrm{Zn}^{2+}$ concentration in a short time frame in the live-cell imaging analysis.

ZnT knockdown. The Silencer Select siRNAs targeting human ZnT5, ZnT6, and ZnT7 were purchased from ThermoFisher Scientific (Supplementary Table 1). The relative amount of mRNA remaining $48 \mathrm{~h}$ after transfection was determined by qRT-PCR analysis using THUNDERBIRD SYBR qPCR Mix (Toyobo) or Sybr Green RT PCR Master Mix (ThermoFisher). The siRNA sequences and the primers used for qRT-PCR are listed in Supplementary Table 2 and Supplementary Table 3. To observe the effects of ZnT knockdown on the localization of ERp44, HeLa cells were transfected with $5 \mathrm{nM}$ each siRNA mixture for $24 \mathrm{~h}$ and transfected with YFPERp44. After additional incubation for $24 \mathrm{~h}$, cells were fixed and counterstained for GM130.

Secretion assay for ERp44 and its clients. To monitor client retention by ERp44 variants, HeLa cells $\left(3.0 \times 10^{5}\right.$ cells $)$ were plated on $60-\mathrm{mm}$ dishes and transfected with $1 \mu \mathrm{g}$ of Erol $\alpha$-Myc and 100-200 ng of YFP-Mock or YFP-ERp44 expressing plasmids. After incubation for $24 \mathrm{~h}$, cells were washed with $37^{\circ} \mathrm{C}$ pre-warmed Opti-MEM twice and incubated in Opti-MEM for $6 \mathrm{~h}$. To concentrate the secreted proteins, conditioned media were collected and clarified by centrifugation. Then, aliquots of supernatants were precipitated with ice-cold $10 \%$ TCA. Precipitants were washed with acetone and dissolved in $1 \times$ SDS-loading buffer. To analyze intracellular proteins, cells remaining on plates were lysed in $1 \times$ SDS-loading buffer and homogenized by sonication. Samples were denatured at $97^{\circ} \mathrm{C}$ for $5 \mathrm{~min}$ and subjected to SDS-PAGE. Protein bands were visualized by immunoblotting analyses. The signal intensity of relevant bands was measured by ImageJ software (NIH) or Image Lab software (Bio-Rad).

To analyze the effect of specific and targeted $\mathrm{Zn}^{2+}$ deprivation, HeLa cells $\left(3.0 \times 10^{5}\right.$ cells) were silenced using siRNAs targeting human ZnT5, ZnT6, and $\mathrm{ZnT7}(20 \mu \mathrm{M}$ each). After $24 \mathrm{~h}$, cells were then transfected with plasmids driving 
the expression of Erol $\alpha-M y c$ or ERAP1-FLAG ( $2 \mu \mathrm{g})$. After further $48 \mathrm{~h}$, cells were washed twice with Opti-MEM preheated at $37^{\circ} \mathrm{C}$ and incubated in the same medium for $4 \mathrm{~h}$. The spent media were analyzed as above. Cells were detached with trypsin, washed and lysed in $1 \% \mathrm{NP}-40,0.1 \% \mathrm{SDS}, 150 \mathrm{mM} \mathrm{NaCl}, 10 \mathrm{mM}$ Tris-HCl, pH 7.4, $20 \mathrm{mM}$ NEM and protease inhibitors. Nuclei and cell debris were discarded by centrifugation. Aliquots of the cell lysates and supernatants were denatured at $97^{\circ} \mathrm{C}$ for $5 \mathrm{~min}$, resolved by $4-12 \%$ gradient precast gels (Invitrogen) under reduced conditions and analyzed by immunoblotting.

Sequential elution assay. HepG2 cells $\left(1.5 \times 10^{6}\right.$ cells $)$ were plated on $10 \mathrm{~cm}$ dishes and transfected with plasmids driving the expression of Halo-RDEL, HaloERp44 WT or Halo-ERp44 3HA, using FuGENE-HD. In some experiments cells were co-transfected also with HA-tagged ERp44 WT or 3HA to better detect 'homo'-oligomerization. After $48 \mathrm{~h}$, cells were lysed in $1 \% \mathrm{NP}-40,150 \mathrm{mM} \mathrm{NaCl}$, $10 \mathrm{mM}$ Tris- $\mathrm{HCl}, \mathrm{pH} 7.4$ for $20 \mathrm{~min}$ on ice and detached by gentle scraping of the culture dishes. Post-nuclear supernatants (PNS) were collected and $1 \mathrm{mg}$ of total protein incubated overnight with immobilized Halo-ligands (Halo-Tagged protein purification resins, Promega). Beads were then washed twice with $150 \mathrm{mM} \mathrm{NaCl}$, $10 \mathrm{mM}$ Tris- $\mathrm{HCl}, \mathrm{pH} 7.5,0.0005 \% \mathrm{NP}-40$, and then sequentially eluted with TPEN $(100 \mu \mathrm{M})$ and, after further washes in the above buffer, eluted with dithiothreitol (DTT, $200 \mathrm{mM}$ ) and $1 \%$ SDS. In case of $\mathrm{Zn}^{2+}$-supplemented conditions, $\mathrm{ZnCl}_{2}$ $(50 \mu \mathrm{M})$ was added to the lysis and wash buffers. Samples were concentrated by TCA precipitation as described above, resuspended in loading buffer, boiled for 5 min and resolved by $4-12 \%$ gradient precast gels under reducing conditions. Proteins of interest were visualized by immunoblotting using antibodies specific for ERp44 (36C9 monoclonal), Halo or HA, as described in figure legends.

GST-GFP-nanobody pull-down assay. GST-GFP-Nanobody proteins were bacterially expressed using BL21 strain and conjugated to glutathione-Sepharose beads. HeLa cells $\left(3.0 \times 10^{5}\right.$ cells) plated on $60-\mathrm{mm}$ plate were transfected with plasmids and cultured for $36 \mathrm{~h}$. Cells were washed with $1 \times$ HHBSS three times and culturing media were exchanged to Opti-MEM containing $2.5 \mu \mathrm{M}$ ZPT or DMSO and incubated for $15 \mathrm{~min}$. Cells were then lysed in lysis buffer $(50 \mathrm{mM}$ HEPES$\mathrm{NaOH}, \mathrm{pH}$ 7.0, $150 \mathrm{mM} \mathrm{NaCl}, 10 \%$ glycerol, $1 \%$ NP-40, 0.1\% SDS, $20 \mathrm{mM}$ NEM and Protease inhibitor cocktail (Nacalai Tesque)). Lysates were homogenized by sonication and clarified by centrifugation and resultant supernatant were precleared by incubation with Protein G Sepharose beads at $4{ }^{\circ} \mathrm{C}$ for $1 \mathrm{~h}$ with rotation. After incubation, beads were precipitated by centrifugation and supernatants were incubated with GST-GFP-Nanobody-conjugated beads at $4{ }^{\circ} \mathrm{C}$ for $3.5 \mathrm{~h}$ with rotation. Then, beads were washed with lysis buffer three times and dissolved by $1 \times$ SDS-loading buffer. Precipitated proteins were denatured at $70{ }^{\circ} \mathrm{C}$ for $10 \mathrm{~min}$ and subjected to SDS-PAGE and immunoblotting analyses.

Gel filtration analysis for microsomal lysates. HEK293T cells from four $15 \mathrm{~cm}$ dishes were cultured to confluence, harvested and homogenized in a buffer containing $10 \mathrm{mM}$ HEPES-NaOH, pH 7.5, $250 \mathrm{mM}$ sucrose, $1 \mathrm{mM}$ EGTA, $0.1 \mathrm{mg} / \mathrm{mL}$ DNaseI, and protease inhibitor cocktail (Nacalai Tesque) by Dounce-type homogenizer until cells were sufficiently disrupted. Unbroken cells and nuclei were removed by three times of centrifugation at $2000 \times \mathrm{g}$ for $10 \mathrm{~min}$. The resultant supernatants (i.e., PNS) were subjected to ultracentrifugation $(100,000 \times g, 1 \mathrm{~h})$ to precipitate microsomes. Microsomes were then resuspended in lysis buffer B (20 $\mathrm{mM}$ BisTris- $\mathrm{HCl}, \mathrm{pH} 7.2,150 \mathrm{mM} \mathrm{NaCl}$ and protease inhibitor cocktail) by passing through $25 \mathrm{G}$ needle and lysed by brief sonication on ice. Lysates were clarified by sequential centrifugation at $21,000 \times g(30 \mathrm{~min})$ and $100,000 \times g(30 \mathrm{~min})$ and filtration through $0.22 \mu \mathrm{m}$ filter. Clarified lysate was loaded onto Superdex 200 Increase $10 / 300$ column equilibrated in $20 \mathrm{mM}$ BisTris- $\mathrm{HCl}, \mathrm{pH} 7.2$ and $150 \mathrm{mM}$ $\mathrm{NaCl}$. The fractions were mixed with NEM (final $1 \mathrm{mM}$ ) and $5 \times$ Laemmli sample buffer and analyzed by immunoblotting. To test the effect of $\mathrm{Zn}^{2+}$ and disulfide bonds, TPEN (final $100 \mu \mathrm{M}$ ) and TCEP (final $1 \mathrm{mM}$ ) was added into PNS and lysis buffer B, respectively.

Statistical analyses. Statistical analyses were performed with Prism software version 7.0a (GraphPad Software), using a two-tailed unpaired $t$-test or one-way ANOVA followed by Dunnett's test or Tukey's test for comparison of multiple datasets. Data represent the means of the indicated number of independent experiments. Error bars indicate the standard error of the mean (SEM) or the standard deviation (SD). $p<0.05$ was considered to be significant.

\section{Data availability}

Coordinates and structure factors have been deposited in the Protein Data Bank with accession code 5XWM. The source data underlying Figs. 1c, 2b, 4c, and $5 c$ and Supplementary Figs. 12A-C, 13B, C, E are provided as a Source Data file. Other data are available from the corresponding authors upon reasonable request.

Received: 23 July 2018 Accepted: 9 January 2019

Published online: 05 February 2019

\section{References}

1. Andreini, C., Banci, L., Bertini, I. \& Rosato, A. Counting the zinc-proteins encoded in the human genome. J. Proteome Res. 5, 196-201 (2006).

2. Andreini, C. \& Bertini, I. A bioinformatics view of zinc enzymes. J. Inorg. Biochem. 111, 150-156 (2012).

3. Auld, D. S. Zinc coordination sphere in biochemical zinc sites. Biometals 14, 271-313 (2001).

4. Patel, K., Kumar, A. \& Durani, S. Analysis of the structural consensus of the zinc coordination centers of metalloprotein structures. Biochim. Biophys. Acta 1774, 1247-1253 (2007).

5. Maret, W. \& Li, Y. Coordination dynamics of zinc in proteins. Chem. Rev. 109, $4682-4707$ (2009).

6. Fukada, T., Yamasaki, S., Nishida, K., Murakami, M. \& Hirano, T. Zinc homeostasis and signaling in health and diseases: zinc signaling. J. Biol. Inorg. Chem. 16, 1123-1134 (2011).

7. Hirano, T. et al. Roles of zinc and zinc signaling in immunity: zinc as an intracellular signaling molecule. Adv. Immunol. 97, 149-176 (2008).

8. Maret, W. Metals on the move: zinc ions in cellular regulation and in the coordination dynamics of zinc proteins. Biometals 24, 411-418 (2011).

9. Jeong, J. \& Eide, D. J. The SLC39 family of zinc transporters. Mol. Asp. Med 34, 612-619 (2013)

10. Huang, L. \& Tepaamorndech, S. The SLC30 family of zinc transporters-a review of current understanding of their biological and pathophysiological roles. Mol. Asp. Med. 34, 548-560 (2013).

11. Kambe, T., Tsuji, T., Hashimoto, A. \& Itsumura, N. The physiological, biochemical, and molecular roles of zinc transporters in zinc homeostasis and metabolism. Physiol. Rev. 95, 749-784 (2015).

12. Kimura, T. \& Kambe, T. The functions of metallothionein and ZIP and ZnT transporters: an overview and perspective. Int. J. Mol. Sci. 17, 336 (2016).

13. Seals, D. F. \& Courtneidge, S. A. The ADAMs family of metalloproteases: multidomain proteins with multiple functions. Genes Dev. 17, 7-30 (2003).

14. Taylor, K. M., Morgan, H. E., Johnson, A. \& Nicholson, R. I. Structurefunction analysis of HKE4, a member of the new LIV-1 subfamily of zinc transporters. Biochem. J. 377, 131-139 (2004).

15. Eide, D. J. Zinc transporters and the cellular trafficking of zinc. Biochim. Biophys. Acta 1763, 711-722 (2006).

16. Fukunaka, A. et al. Tissue nonspecific alkaline phosphatase is activated via a two-step mechanism by zinc transport complexes in the early secretory pathway. J. Biol. Chem. 286, 16363-16373 (2011).

17. Jeong, J. et al. Promotion of vesicular zinc efflux by ZIP13 and its implication for spondylocheiro dysplastic Ehlers-Danlos syndrome. Proc. Natl Acad. Sci. USA 109, E3530-E3538 (2012).

18. Kambe, T., Takeda, T. A. \& Nishito, Y. Activation of zinc-requiring ectoenzymes by $\mathrm{ZnT}$ transporters during the secretory process: Biochemical and molecular aspects. Arch. Biochem. Biophys. 611, 37-42 (2016).

19. Tsuji, T. et al. Dissecting the process of activation of cancer-promoting zincrequiring ectoenzymes by zinc metalation mediated by ZNT transporters. $J$. Biol. Chem. 292, 2159-2173 (2017).

20. Anelli, T., Sannino, S. \& Sitia, R. Proteostasis and "redoxtasis" in the secretory pathway: tales of tails from ERp44 and immunoglobulins. Free Radic. Biol. Med. 83, 323-330 (2015).

21. Anelli, T. \& Sitia, R. Protein quality control in the early secretory pathway. EMBO J. 27, 315-327 (2008).

22. Otsu, M. et al. Dynamic retention of Erolalpha and Erolbeta in the endoplasmic reticulum by interactions with PDI and ERp44. Antioxid. Redox Signal. 8, 274-282 (2006)

23. Fraldi, A. et al. Multistep, sequential control of the trafficking and function of the multiple sulfatase deficiency gene product, SUMF1 by PDI, ERGIC- 53 and ERp44. Hum. Mol. Genet. 17, 2610-2621 (2008).

24. Kakihana, T. et al. Dynamic regulation of Erolalpha and peroxiredoxin 4 localization in the secretory pathway. J. Biol. Chem. 288, 29586-29594 (2013).

25. Hisatsune, C. et al. ERp44 exerts redox-dependent control of blood pressure at the ER. Mol. Cell 58, 1015-1027 (2015).

26. Yang, K. et al. Crystal structure of the ERp44-peroxiredoxin 4 complex reveals the molecular mechanisms of thiol-mediated protein retention. Structure 24, 1755-1765 (2016).

27. Anelli, T. et al. Thiol-mediated protein retention in the endoplasmic reticulum: the role of ERp44. EMBO J. 22, 5015-5022 (2003).

28. Anelli, T. et al. Sequential steps and checkpoints in the early exocytic compartment during secretory IgM biogenesis. EMBO J. 26, 4177-4188 (2007).

29. Wang, Z. V. et al. Secretion of the adipocyte-specific secretory protein adiponectin critically depends on thiol-mediated protein retention. Mol. Cell. Biol. 27, 3716-3731 (2007).

30. Qiang, L., Wang, H. \& Farmer, S. R. Adiponectin secretion is regulated by SIRT1 and the endoplasmic reticulum oxidoreductase Ero1-L alpha. Mol. Cell. Biol. 27, 4698-4707 (2007). 
31. Hampe, L. et al. Regulation and quality control of adiponectin assembly by endoplasmic reticulum chaperone ERp44. J. Biol. Chem. 290, 18111-18123 (2015).

32. Vavassori, S. et al. A pH-regulated quality control cycle for surveillance of secretory protein assembly. Mol. Cell 50, 783-792 (2013).

33. Wang, L. et al. Crystal structure of human ERp44 shows a dynamic functional modulation by its carboxy-terminal tail. EMBO Rep. 9, 642-647 (2008).

34. Watanabe, S., Harayama, M., Kanemura, S., Sitia, R. \& Inaba, K. Structural basis of $\mathrm{pH}$-dependent client binding by ERp44, a key regulator of protein secretion at the ER-Golgi interface. Proc. Natl Acad. Sci. USA 114, E3224-E3232 (2017).

35. Okumura, M., Watanabe, S. \& Inaba, K. in Oxidative Folding of Proteins: Basic Principles, Cellular Regulation and Engineering (ed. Feige, M. J.) 224-248 (The Royal Society of Chemistry, 2018).

36. Anelli, T. \& Sitia, R. in Oxidative Folding of Proteins: Basic Principles, Cellular Regulation and Engineering (ed. Feige, M. J.) 249-266 (The Royal Society of Chemistry, 2018)

37. Sannino, S. et al. Progressive quality control of secretory proteins in the early secretory compartment by ERp44. J. Cell. Sci. 127, 4260-4269 (2014).

38. Wang, L. et al. Reconstitution of human Erol-Lalpha/protein-disulfide isomerase oxidative folding pathway in vitro. Position-dependent differences in role between the a and a' domains of protein-disulfide isomerase. J. Biol. Chem. 284, 199-206 (2009).

39. Masui, S., Vavassori, S., Fagioli, C., Sitia, R. \& Inaba, K. Molecular bases of cyclic and specific disulfide interchange between human EROlalpha protein and protein-disulfide isomerase (PDI). J. Biol. Chem. 286, 16261-16271 (2011).

40. Polishchuk, R. \& Lutsenko, S. Golgi in copper homeostasis: a view from the membrane trafficking field. Histochem. Cell. Biol. 140, 285-295 (2013).

41. He, W. \& Hu, Z. The role of the Golgi-resident SPCA Ca $(2)(+) / \mathrm{Mn}(2)(+)$ pump in ionic homeostasis and neural function. Neurochem. Res. 37, 455-468 (2012).

42. Maret, W. New perspectives of zinc coordination environments in proteins. J. Inorg. Biochem. 111, 110-116 (2012).

43. Zastrow, M. L. \& Pecoraro, V. L. Designing hydrolytic zinc metalloenzymes. Biochemistry 53, 957-978 (2014).

44. King, D. A., Zhang, L., Guarente, L. \& Marmorstein, R. Structure of a HAP1DNA complex reveals dramatically asymmetric DNA binding by a homodimeric protein. Nat. Struct. Biol. 6, 64-71 (1999).

45. Dhakshnamoorthy, B., Mizuno, H. \& Kumar, P. K. Alternative binding modes of l-histidine guided by metal ions for the activation of the antiterminator protein HutP of Bacillus subtilis. J. Struct. Biol. 183, 512-518 (2013).

46. Andreini, C., Cavallaro, G., Lorenzini, S. \& Rosato, A. MetalPDB: a database of metal sites in biological macromolecular structures. Nucleic Acids Res. 41, D312-D319 (2013).

47. Peters, M. B. et al. Structural survey of zinc containing proteins and the development of the zinc AMBER force field (ZAFF). J. Chem. Theory Comput. 6, 2935-2947 (2010).

48. Dam, T. K., Roy, R., Page, D. \& Brewer, C. F. Negative cooperativity associated with binding of multivalent carbohydrates to lectins. Thermodynamic analysis of the "multivalency effect". Biochemistry 41, 1351-1358 (2002).

49. Martinez, J. C. et al. in Applications of Calorimetry in a Wide Context: Differential Scanning Calorimetry, Isothermal Titration Calorimetry and Microcalorimeter (ed. Elkordy, A. A.) (InTech, Rijeka, Croatia, 2013).

50. Anelli, T. et al. ERp44, a novel endoplasmic reticulum folding assistant of the thioredoxin family. EMBO J. 21, 835-844 (2002).

51. Coyle, P., Philcox, J. C., Carey, L. C. \& Rofe, A. M. Metallothionein: the multipurpose protein. Cell. Mol. Life Sci. 59, 627-647 (2002).

52. Krezel, A. \& Maret, W. Zinc-buffering capacity of a eukaryotic cell at physiological pZn. J. Biol. Inorg. Chem. 11, 1049-1062 (2006).

53. McRae, R., Lai, B. \& Fahrni, C. J. Subcellular redistribution and mitotic inheritance of transition metals in proliferating mouse fibroblast cells. Metallomics 5, 52-61 (2013).

54. McCormick, N., Velasquez, V., Finney, L., Vogt, S. \& Kelleher, S. L. X-ray fluorescence microscopy reveals accumulation and secretion of discrete intracellular zinc pools in the lactating mouse mammary gland. PLoS ONE 5, e11078 (2010).

55. Ohana, E. et al. Identification of the $\mathrm{Zn} 2+$ binding site and mode of operation of a mammalian Zn2+ transporter. J. Biol. Chem. 284, 17677-17686 (2009).

56. Qin, Y., Dittmer, P. J., Park, J. G., Jansen, K. B. \& Palmer, A. E. Measuring steady-state and dynamic endoplasmic reticulum and Golgi $\mathrm{Zn} 2+$ with genetically encoded sensors. Proc. Natl Acad. Sci. USA 108, 7351-7356 (2011).

57. Chabosseau, P. et al. Mitochondrial and ER-targeted eCALWY probes reveal high levels of free Zn2+. ACS Chem. Biol. 9, 2111-2120 (2014).

58. Hessels, A. M. et al. eZinCh-2: a versatile, genetically encoded FRET sensor for cytosolic and intraorganelle $\mathrm{Zn}(2+)$ imaging. ACS Chem. Biol. 10, 2126-2134 (2015).

59. Hessels, A. M., Taylor, K. M. \& Merkx, M. Monitoring cytosolic and ER $\mathrm{Zn}(2+)$ in stimulated breast cancer cells using genetically encoded FRET sensors. Metallomics 8, 211-217 (2016).
60. Homma, K. et al. SOD1 as a molecular switch for initiating the homeostatic ER stress response under zinc deficiency. Mol. Cell 52, 75-86 (2013).

61. Ishihara, K. et al. Zinc transport complexes contribute to the homeostatic maintenance of secretory pathway function in vertebrate cells. J. Biol. Chem. 281, 17743-17750 (2006).

62. Renna, M., Caporaso, M. G., Bonatti, S., Kaufman, R. J. \& Remondelli, P. Regulation of ERGIC-53 gene transcription in response to endoplasmic reticulum stress. J. Biol. Chem. 282, 22499-22512 (2007).

63. Munro, S. \& Pelham, H. R. A C-terminal signal prevents secretion of luminal ER proteins. Cell 48, 899-907 (1987).

64. Gilchrist, A. et al. Quantitative proteomics analysis of the secretory pathway. Cell 127, 1265-1281 (2006).

65. Choi, S. \& Bird, A. J. Zinc'ing sensibly: controlling zinc homeostasis at the transcriptional level. Metallomics 6, 1198-1215 (2014)

66. Gunther, V., Lindert, U. \& Schaffner, W. The taste of heavy metals: gene regulation by MTF-1. Biochim. Biophys. Acta 1823, 1416-1425 (2012).

67. Maret, W. Inhibitory zinc sites in enzymes. Biometals 26, 197-204 (2013).

68. Wilson, M., Hogstrand, C. \& Maret, W. Picomolar concentrations of free zinc (II) ions regulate receptor protein-tyrosine phosphatase beta activity. J. Biol. Chem. 287, 9322-9326 (2012).

69. Anderson, C. T. et al. Modulation of extrasynaptic NMDA receptors by synaptic and tonic zinc. Proc. Natl Acad. Sci. USA 112, E2705-E2714 (2015).

70. Ronzoni, R. et al. Pathogenesis of ER storage disorders: modulating Russell body biogenesis by altering proximal and distal quality control. Traffic 11, 947-957 (2010).

71. Benham, A. M. et al. The CXXCXXC motif determines the folding, structure and stability of human Erol-Lalpha. EMBO J. 19, 4493-4502 (2000)

72. Keller, S. et al. High-precision isothermal titration calorimetry with automated peak-shape analysis. Anal. Chem. 84, 5066-5073 (2012).

73. Zhao, H., Piszczek, G. \& Schuck, P. SEDPHAT-a platform for global ITC analysis and global multi-method analysis of molecular interactions. Methods 76, 137-148 (2015)

74. Brautigam, C. A., Zhao, H., Vargas, C., Keller, S. \& Schuck, P. Integration and global analysis of isothermal titration calorimetry data for studying macromolecular interactions. Nat. Protoc. 11, 882-894 (2016).

75. Kabsch, W. Xds. Acta Crystallogr. D Biol. Crystallogr. 66, 125-132 (2010).

76. Sheldrick, G. M. Experimental phasing with SHELXC/D/E: combining chain tracing with density modification. Acta Crystallogr. D Biol. Crystallogr. 66, 479-485 (2010).

77. Cowtan, K. The Buccaneer software for automated model building. 1. Tracing protein chains. Acta Crystallogr. D Biol. Crystallogr. 62, 1002-1011 (2006).

78. Emsley, P. \& Cowtan, K. Coot: model-building tools for molecular graphics. Acta Crystallogr. D Biol. Crystallogr. 60, 2126-2132 (2004).

79. Adams, P. D. et al. PHENIX: a comprehensive Python-based system for macromolecular structure solution. Acta Crystallogr. D Biol. Crystallogr. 66, 213-221 (2010).

80. Chen, V. B. et al. MolProbity: all-atom structure validation for macromolecular crystallography. Acta Crystallogr. D Biol. Crystallogr. 66, 12-21 (2010).

81. Pettersen, E. F. et al. UCSF Chimera--a visualization system for exploratory research and analysis. J. Comput. Chem. 25, 1605-1612 (2004).

82. Miranda, J. G. et al. New alternately colored FRET sensors for simultaneous monitoring of $\mathrm{Zn}(2)(+)$ in multiple cellular locations. PLoS ONE 7, e49371 (2012).

\section{Acknowledgements}

We would like to thank K. Nakayama for providing pGEX6P1-GFP-Nanobody; $\mathrm{H}$ Kadokura for providing pcDNA3.1-Erol $\alpha-M y c ; K$. Miki for assistance with SEC-MALS experiments; S. Takahashi for help with CD measurements, S. Ogawa for her help with sample preparation; the beamline scientists of SPring- 8 and the Photon Factory for their help with the X-ray data collection; Claudio Fagioli and Andrea Orsi for invaluable technical help, ALEMBIC (OSR-Milan-Italy) and in particular Valeria Berno for help with IF acquisition and A. de Matteis (TIGEM, Naples, Italy) for providing excellent antiGM130 polyclonal antibodies. R.S. thanks Annalisa Pastore for raising his attention on zinc. This work was supported by CREST (to K.I.), Grants-in-Aid for Scientific Research (15H04335, 18H03978 and 26116005 to K.I., 18K06075 to S.W., and 17K15085 to Y.A.) from the MEXT of Japan, the Platform Project for Supporting in Drug Discovery and Life Science Research (Platform for Drug Discovery, Informatics, and Structural Life Science) from Japan Agency for Medical Research and Development (AMED) (to K.I) and Telethon (GGP15059), Associazione Italiana per la Ricerca sul Cancro (AIRC, IG 14559) and Fondazione Cariplo (2015-0591) to R.S. S.S. acknowledges the receipt of a long-term EMBO post-doctoral fellowship (ALTF 823-2016).

\section{Author contributions}

S.W., Y.A., S.S., R.S., and K.I. designed the research. S.W., M.H., and S.M. performed in vitro biochemical analyses. S.W. and M.H. performed crystallization and structure determination. Y.A., S.S., T.T., and T.A. performed in vivo assays. Y.A., M.Y., I.S., and 
T.A. performed immunofluorescence analyses. Y.A. performed live-cell imaging. S.W., Y.A., and S.S. wrote the initial draft. S.W, Y.A, S.S., T.A., M.H., R.S., and K.I. wrote the manuscript. K.I. and R.S. supervised the research.

\section{Additional information}

Supplementary Information accompanies this paper at https://doi.org/10.1038/s41467019-08429-1.

Competing interests: The authors declare no competing interests.

Reprints and permission information is available online at http://npg.nature.com/ reprintsandpermissions/

Journal peer review information Nature Communications thanks Ángel Pérez-Lara, Yan Qin and the other anonymous reviewer(s) for their contribution to the peer review of this work. Peer reviewer reports are available.
Publisher's note: Springer Nature remains neutral with regard to jurisdictional claims in published maps and institutional affiliations.

(c) (i) Open Access This article is licensed under a Creative Commons Attribution 4.0 International License, which permits use, sharing, adaptation, distribution and reproduction in any medium or format, as long as you give appropriate credit to the original author(s) and the source, provide a link to the Creative Commons license, and indicate if changes were made. The images or other third party material in this article are included in the article's Creative Commons license, unless indicated otherwise in a credit line to the material. If material is not included in the article's Creative Commons license and your intended use is not permitted by statutory regulation or exceeds the permitted use, you will need to obtain permission directly from the copyright holder. To view a copy of this license, visit http://creativecommons.org/ licenses/by/4.0/.

(C) The Author(s) 2019 